\title{
THE INVERSE GALOIS PROBLEM AND
}

\section{RATIONAL POINTS ON MODULI SPACES}

\author{
Michael D. Fried*, UC Irvine \\ Helmut Völklein**, $U$ of Florida and Universität Erlangen
}

\begin{abstract}
We reduce the regular version of the Inverse Galois Problem for any finite group $G$ to finding one rational point on an infinite sequence of algebraic varieties. As a consequence, any finite group $G$ is the Galois group of an extension $L / P(x)$ with $L$ regular over any PAC field $P$ of characteristic zero. A special case of this implies that $G$ is a Galois group over $\mathcal{F}_{p}(x)$ for almost all primes $p$.
\end{abstract}

\section{§. INTRODUCTION}

Many attempts have been made to realize finite groups as Galois groups of extensions of $\mathcal{Q}(x)$ that are regular over $\mathcal{Q}$ (see the end of this introduction for definitions). We call this the "regular inverse Galois problem." We show that to each finite group $G$ with trivial center and integer $r \geq 3$ there is canonically associated an algebraic variety, $\mathcal{H}_{r}^{\text {in }}(G)$, defined over $\mathcal{Q}$ (usually reducible) satisfying the following.

Fundamental Property: There exists a Galois extension of $\mathcal{Q}(x)$, regular over $\mathcal{Q}$, with Galois group isomorphic to $G$ and with $r$ branch points, if and only if $\mathcal{H}_{r}^{\text {in }}(G)$ has a $\mathcal{Q}$-rational point. (This holds if $\mathcal{Q}$ is replaced by any field of characteristic 0 ).

This is contained in Corollary 1 (in $\S 2.1$ ). Theorem 1, our main result, gives a more general formulation that applies to any finite group. The Fundamental Property reduces the regular inverse Galois problem to finding $\mathcal{Q}$-points on certain (reducible) varieties. The first step towards this is to show that (under certain conditions) there exist absolutely irreducible components of $\mathcal{H}_{r}^{\text {in }}(G)$ that are defined over $\mathcal{Q}$. The components of $\mathcal{H}_{r}^{\text {in }}(G)$ are in one-one correspondence with the orbits of the Hurwitz monodromy group in its action on certain classes of generating systems of $G$ (see $\S 1.3$ ).

*Supported by NSF grant DMS-8702150 and BSF grant \#87-00038

** Supported by NSA grant MDA 904-89-H-2028

AMS Subject classification: 11G35, 12F10, 14D20, 14E20, 14G05, 20B25, 20C25

Keywords: Riemann's existence theorem; Galois groups; Nielsen classes; Braid and Hurwitz monodromy groups; PAC-fields. 
Using a theorem of Conway and Parker $[\mathrm{CP}]$ on such group actions, we conclude that the space $\mathcal{H}_{r}^{\text {in }}(G)$ has an (absolutely) irreducible component defined over $\mathcal{Q}$ if we allow $r$ to be large and replace $G$ by some group with quotient $G$ (see $\S 2.2$ ). The $\mathcal{Q}$-components that we construct this way are generalizations of the classical Hurwitz spaces.

It is convenient to introduce the following terminology: A group $G$ is called regular over a field $k$ if $G$ is isomorphic to the Galois group of an extension of $k(x)$ that is regular over $k$. The above has the following immediate corollary for $\mathrm{P}$ (seudo)A(lgebraically)C(losed) fields $P$ of characteristic 0: Every finite group is regular over $P$ (Theorem 2). Another corollary (which can be viewed as a special case of the previous one) is that every finite group is regular over the finite prime field $\mathcal{F}_{p}$ for almost all primes $p$ (Corollary 2).

In $\S 6$ we derive an addendum to our main result that is crucial for the preprint [FrVo]. In that paper we prove a long-standing conjecture on Hilbertian PAC-fields $P$ (in the case $\operatorname{char}(P)=0$ ): Every finite embedding problem over $P$ is solvable. For countable $P$ this, combined with a result of Iwasawa, implies that the absolute Galois group of $P$ is $\omega$-free. That is, $G(\bar{P} / P)$ is a free profinite group of countably infinite rank, denoted $\hat{F}_{\omega}$. By a result of [FrJ, 2], every countable Hilbertian field $k$ of characteristic 0 has a Galois extension $P$ with the following properties: $P$ is Hilbertian and PAC, and $G(P / k) \cong \prod_{n=2}^{\infty} S_{n}$ (where $S_{n}$ is the symmetric group of degree $n)$. From the above, $G(\bar{k} / P)=G(\bar{P} / P) \cong \hat{F}_{\omega}$, and we get the exact sequence

$$
1 \rightarrow \hat{F}_{\omega} \rightarrow G(\bar{k} / k) \rightarrow \prod_{n=2}^{\infty} S_{n} \rightarrow 1 .
$$

Moduli spaces for branched covers of $\mathcal{P}^{1}$ were already considered by Hurwitz [Hur] in the special case of simple branching (where the Galois group is $S_{n}$ ). Fulton [Fu] showed - still in the case of simple branching - that the analytic moduli spaces studied by Hurwitz are the sets of complex points of certain schemes. Fried [Fr,1] studied more generally moduli spaces for covers of $\mathcal{P}^{1}$ with an arbitrary given monodromy group $G \subset S_{n}$ and with a fixed number of branch points. The new moduli spaces $\mathcal{H}_{r}^{\text {in }}(G)$ studied in the present paper are coverings of those previous ones, parametrizing equivalence classes of pairs $(\chi, h)$ where $\chi$ is a Galois cover of $\mathcal{P}^{1}$ with $r$ branch points and $h$ is an isomorphism between $G$ and the automorphism group of the cover $\chi$. The extra data of the isomorphism $h$ associated to the points of $\mathcal{H}_{r}^{\text {in }}(G)$ ensures that a $\mathcal{Q}$ rational point corresponds to a cover of $\mathcal{P}^{1}$ that can be defined over $\mathcal{Q}$ such that also all its automorphisms are defined over $\mathcal{Q}$; the latter condition guarantees that the corresponding function field extension $L / \mathcal{Q}(x)$ is Galois with group $G$.

The proof of our main result relies on the construction of a family of certain covers of $\mathcal{P}^{1}$ with no (non-trivial) automorphisms $(\S 4)$. Then we use the interplay between the spaces $\mathcal{H}_{r}^{\text {in }}(G)$ and moduli spaces $\mathcal{H}_{r}^{\mathrm{ab}}(G, U)$ of equivalence classes of certain covers that are not Galois. Matzat [Ma, 2] has studied the function fields of the absolutely irreducible components of the spaces $\mathcal{H}_{r}^{\text {in }}(G)$ and $\mathcal{H}_{r}^{\text {ab }}(G, U)$ (for $U=1$ ) from another point of view (using profinite algebraic fundamental groups).

Because of the essential use we make of the unpublished result [CP] (in the applications of our main result), we supply an appendix where we give a (slightly modified and corrected) proof of the version of [CP] that is needed here.

Acknowledgements: Moshe Jarden pointed out many of the potential consequences of the Main Theorem to the first author.

Notations: Throughout $x$ will denote an indeterminate that is transcendental over any particular base field, usually denoted $k$. Thus $k(x)$ is the field of rational functions in $x$ with coefficients in $k$. The algebraic closure of a field $k$ is denoted by $\bar{k}$, and we let $G_{k}=G(\bar{k} / k)$ denote the absolute Galois group of $k$ (in characteristic 0 ). A field $L$ is said to be regular over a subfield $k$ if $L$ and $\bar{k}$ are linearly disjoint over $k$; in the case $\operatorname{char}(L)=0$ this is equivalent to the condition that $k$ is algebraically closed in $L$. We say that a finite group $G$ is regular over a field $k$ (with $r$ branch points) if $G$ is isomorphic to the Galois group of an extension $L / k(x)$ with $L$ regular over $k$ (and with $r$ branch points). When we speak of the branch points of such an extension $L / k(x)$ (with $\operatorname{char}(k)=0$ ), we mean branch points of the corresponding curve cover over $\bar{k}$. 
The field of rationals (resp., complexes) is denoted by $\mathcal{Q}$ (resp., $\mathcal{C}$ ). We let $\mathcal{P}^{1}$ denote the Riemann sphere $\mathcal{C} \cup\{\infty\}$, viewed according to context as a Riemann surface or as an algebraic curve defined over $\mathcal{Q}$ (in the natural way). The fundamental group of a topological space $Y$, based at $y \in Y$, is denoted $\pi_{1}(Y, y)$. If $U$ is a subgroup of $G$, then $[U]$ denotes the conjugacy class of subgroups of $G$ containing $U$. A subgroup of $G$ is called self-normalizing if it equals its own normalizer in $G$.

\section{TABLE OF CONTENTS}

\section{§0. INTRODUCTION}

\section{§1. MODULI SPACES FOR COVERS OF THE RIEMANN SPHERE}

$\S 1.1$. Nielsen classes and the Hurwitz monodromy group

$\S 1.2$. Moduli spaces for Covers of $\mathcal{P}^{1}$

§1.3. Monodromy Action on the fibers of $\mathcal{H}^{\text {ab }}$ and $\mathcal{H}^{\text {in }}$

$\S 1.4$. The action of $Q_{i}$ on $\mathcal{E}_{r}^{\text {ab }}$ and $\mathcal{E}_{r}^{\text {in }}$

$\S 1.5$. The field of definition of a cover

\section{§. THE MAIN THEOREM AND SOME CONSEQUENCES}

$\S 2.1$. The Main Theorem

$\S 2.2$. Irreducibility of Hurwitz spaces, and full high branching

$\S 2.3$. The application to PAC-fields

$\S 2.4$. Two group-theoretic Lemmas

\section{§3. PROOF OF THEOREM 1 UNDER A CONTINUITY ASSUMP-} TION

§3.1. The $\mathcal{Q}$-structure on $\mathcal{H}^{\text {ab }}$ and $\mathcal{H}^{\text {in }}$

$\S 3.2$. The remaining part of Theorem 1

\section{§4. FAMILIES OF COVERS}

$\S 4.1$. The topological construction of the family

$\S 4.2$. Uniqueness of the family

$\S 4.3$. The $\epsilon_{\beta}$ are continuous

\section{$\S 5$. CONCLUSION OF THE PROOF OF THEOREM 1}

$\S 5.1$. Some reductions

§5.2. The $\epsilon_{\beta}^{\prime}$ are continuous

§5.3. Another group-theoretic Lemma

\section{§6. A RESULT FOR LATER USE}

$\S 6.1$. The automorphisms $\delta_{A}$ of $\mathcal{H}^{\text {in }}$ over $\mathcal{H}^{\text {ab }}$

$\S 6.2$. Choosing suitable Hurwitz spaces

$\S 6.3$. The main result of $\S 6$

APPENDIX The Conway-Parker Theorem
A. Introduction
B. Central Extensions
C. Congruence Classes of Words 


\section{MODULI SPACES FOR COVERS OF THE RIEMANN SPHERE}

Let $G$ be a finite group and $U \leq G$ a subgroup that does not contain a non-trivial normal subgroup of $G$. Let $\operatorname{Aut}(G, U)$ be the group of all automorphisms of $G$ that preserve the conjugacy class of $U$, and let $\operatorname{Inn}(G)$ be the group of inner automorphisms.

$\S 1.1$. Nielsen classes and the Hurwitz monodromy group: In this subsection we fix some more terminology. Let $r$ be an integer $>1$, and consider the set

$$
\mathcal{E}_{r}=\mathcal{E}_{r}(G)=\left\{\left(\sigma_{1}, \ldots, \sigma_{r}\right) \mid \sigma_{1}, \ldots, \sigma_{r} \in G \backslash\{1\},<\sigma_{1}, \ldots, \sigma_{r}>=G, \sigma_{1} \cdots \sigma_{r}=1\right\}
$$

Let $\mathcal{E}_{r}^{\mathrm{ab}}=\mathcal{E}_{r}^{\mathrm{ab}}(G, U)$ (resp., $\mathcal{E}_{r}^{\mathrm{in}}=\mathcal{E}_{r}^{\mathrm{in}}(G)$ ) denote the quotient of $\mathcal{E}_{r}$ by the componentwise action of $\operatorname{Aut}(G, U)$ (resp., of $\operatorname{Inn}(G))$. For any conjugacy class $\mathrm{C}$ of $G$ and for any integer $m$ define $\mathrm{C}^{m}$ to be the conjugacy class of the $g^{m}, g \in C$. Let $\mathbf{C}=\left(\mathrm{C}_{1}, \ldots, \mathrm{C}_{r}\right)$ be an $r$-tuple of conjugacy classes of $G$. We say $\left(\mathrm{C}_{1}, \ldots, \mathrm{C}_{r}\right)$ is rational if for each integer $m$ prime to the order of $G$ we have $\left(\mathrm{C}_{1}^{m}, \ldots, \mathrm{C}_{r}^{m}\right)=$ $\left(\mathrm{C}_{\pi(1)}, \ldots, \mathrm{C}_{\pi(r)}\right)$ for some $\pi \in S_{r}$. This generalizes the usual notion of a rational conjugacy class.

The Nielsen class $\mathrm{Ni}(\mathbf{C})$ of $\mathbf{C}$ is defined to be the set of all $\left(\sigma_{1}, \ldots, \sigma_{r}\right) \in \mathcal{E}_{r}$ for which there exists a permutation $\pi \in S_{r}$ with $\sigma_{i} \in C_{\pi(i)}$ for $i=1, \ldots, r$. Define the set $\mathrm{Ni}(\mathbf{C})^{\text {ab }}$ (resp., $\left.\mathrm{Ni}(\mathbf{C})^{\text {in }}\right)$ to be the image of $\mathrm{Ni}(\mathbf{C})$ in $\mathcal{E}_{r}^{\text {ab }}$ (resp., $\mathcal{E}_{r}^{\text {in }}$ ).

Embed affine space $\mathcal{A}^{r}$ in $\mathcal{P}^{r}$ by regarding $\mathcal{A}^{r}$ as the space of monic complex polynomials of degree $r$, and $\mathcal{P}^{r}$ as the space of all nonzero complex polynomials of degree at most $r$ up to multiplication by a nonzero constant. Consider the classical discriminant locus in $\mathcal{A}^{r}$, corresponding to the polynomials with repeated roots, and denote its closure in $\mathcal{P}^{r}$ by $D_{r}$. We will work with the space $\mathcal{U}_{r} \stackrel{\text { def }}{=} \mathcal{P}^{r} \backslash D_{r}$, which we view as the space of all subsets of cardinality $r$ of the Riemann sphere $\mathcal{P}^{1}=\mathcal{C} \cup\{\infty\}$. That is, we identify a point of $\mathcal{U}_{r}$ with the set of roots of a corresponding polynomial, where we count $\infty$ as a root if the degree of the polynomial is less than $r$ (the degree is then necessarily $r-1$ ).

The space $\mathcal{U}_{r}$ has a natural structure as algebraic variety defined over $\mathcal{Q}$. We fix a base point $\boldsymbol{b}=$ $\left\{b_{1}, \ldots, b_{r}\right\}$ in $\mathcal{U}_{r}$ that is rational over $\mathcal{Q}$ (i.e., the $b_{i}$ 's are permuted by $G_{\mathcal{Q}}$ ); further we assume $b_{i} \neq \infty$ for all $i$. For the moment we consider $\mathcal{U}_{r}$ only as a complex manifold. The (topological) fundamental group $H_{r}=\pi_{1}\left(\mathcal{U}_{r}, \boldsymbol{b}\right)$ is called the Hurwitz monodromy group (cf. [BF]). It is a quotient of $\pi_{1}\left(\mathcal{A}^{r} \backslash D_{r}, \boldsymbol{b}\right)($ via the map induced from the embedding of $\mathcal{A}^{r}$ in $\mathcal{P}^{r}$ ). The latter group is classically known to be isomorphic to the Artin braid group $B_{r}$. Thus the "elementary braids" that generate $B_{r}$ yield generators $Q_{1}, \ldots, Q_{r-1}$ of $H_{r}$. In $\S 1.3$ we will work with an explicit description of these generators.

$\S 1.2$. Moduli Spaces for Covers of $\mathcal{P}^{1}:$ From now on $\varphi: X \rightarrow \mathcal{P}^{1}$ will always denote a (branched) cover of compact (connected) Riemann surfaces. Two such covers $\varphi: X \rightarrow \mathcal{P}^{1}$ and $\varphi^{\prime}: X^{\prime} \rightarrow \mathcal{P}^{1}$ are called equivalent if there exists an isomorphism $\delta: X \rightarrow X^{\prime}$ with $\varphi^{\prime} \circ \delta=\varphi$. We let $\operatorname{Aut}\left(X / \mathcal{P}^{1}\right)$ denote the group of automorphisms of the cover $\varphi: X \rightarrow \mathcal{P}^{1}$ (i.e., automorphisms $\delta$ of $X$ with $\varphi \circ \delta=\varphi$ ). The cover $\varphi$ is called a Galois cover if $\operatorname{Aut}\left(X / \mathcal{P}^{1}\right)$ is transitive on the fibers of $\varphi$.

Let $a_{1}, \ldots, a_{r} \in \mathcal{P}^{1}$ be the branch points of the cover $\varphi$, and set $\boldsymbol{a}=\left\{a_{1}, \ldots, a_{r}\right\}$. Then $\varphi$ restricts to an (unramified) topological cover $\varphi^{0}$ of the punctured sphere $\mathcal{P}^{1} \backslash \boldsymbol{a}$. Choose a base point $a_{0}$ on this punctured sphere. By the theory of covering spaces, the equivalence class of $\varphi^{0}$ corresponds to a conjugacy class $\left[U_{\varphi}\right]$ of subgroups $U_{\varphi}$ of the fundamental group $\Gamma=\pi_{1}\left(\mathcal{P}^{1} \backslash \boldsymbol{a}, a_{0}\right)$. In fact we have a 1-1 correspondence between the equivalence classes of covers $\varphi^{\prime}: X^{\prime} \rightarrow \mathcal{P}^{1}$ with branch points among $a_{1}, \ldots, a_{r}$, and conjugacy classes of subgroups of $\Gamma$ of finite index (see e.g. $[\mathrm{Fu}, 1.3]$ ). Under this correspondence, the covers with exactly $r$ branch points correspond to those subgroups of $\Gamma$ that do not contain the kernel of the natural map from $\Gamma$ to $\Gamma_{i} \stackrel{\text { def }}{=} \pi_{1}\left(\left(\mathcal{P}^{1} \backslash \boldsymbol{a}\right) \cup\left\{a_{i}\right\}, a_{0}\right)$, for any $i$. Furthermore, under the above correspondence the group $\operatorname{Aut}\left(X / \mathcal{P}^{1}\right)$ is isomorphic to $N_{\Gamma}\left(U_{\varphi}\right) / U_{\varphi}$ where $N_{\Gamma}\left(U_{\varphi}\right)$ is the normalizer of $U_{\varphi}$ in $\Gamma$.

Let $\mathcal{H}^{\mathrm{ab}}=\mathcal{H}_{r}^{\mathrm{ab}}(G, U)$ be the set of equivalence classes $|\varphi|$ of all covers $\varphi: X \rightarrow \mathcal{P}^{1}$ with $r$ branch points for which — in the above notation — there is a surjection $f: \Gamma \rightarrow G$ with $f^{-1}(U)$ conjugate to $U_{\varphi}$. (This is clearly independent of the choice of base point $a_{0}$ ). The 1-1 correspondence between equivalence classes of the covers $\varphi$ and of their restrictions $\varphi^{0}$ allows us to say that the point $|\varphi|$ of $\mathcal{H}^{\text {ab }}$ is represented by $\varphi^{0}$ (as well as by $\varphi$ ). From the last paragraph: 
(1) $\operatorname{Aut}\left(X / \mathcal{P}^{1}\right)=1$ if the class of $\varphi: X \rightarrow \mathcal{P}^{1}$ belongs to $\mathcal{H}^{\mathrm{ab}}=\mathcal{H}_{r}^{\mathrm{ab}}(G, U)$ and $U$ is self-normalizing in $G$.

Let $\mathcal{H}^{\text {in }}=\mathcal{H}_{r}^{\text {in }}(G)$ be the set of equivalence classes of pairs $(\chi, h)$ where $\chi: \hat{X} \rightarrow \mathcal{P}^{1}$ is a Galois cover with $r$ branch points, and $h: \operatorname{Aut}\left(\hat{X} / \mathcal{P}^{1}\right) \rightarrow G$ is an isomorphism; two such pairs $(\chi, h)$ and $\left(\chi^{\prime}: \hat{X}^{\prime} \rightarrow \mathcal{P}^{1}, h^{\prime}\right)$ are called equivalent if there is an isomorphism $\delta: \hat{X} \rightarrow \hat{X}^{\prime}$ over $\mathcal{P}^{1}$ such that $h^{\prime} \circ c_{\delta}=h$, where $c_{\delta}$ : $\operatorname{Aut}\left(\hat{X} / \mathcal{P}^{1}\right) \rightarrow \operatorname{Aut}\left(\hat{X}^{\prime} / \mathcal{P}^{1}\right)$ is the isomorphism induced by $\delta$. Let $|\chi, h|$ denote the equivalence class of the pair $(\chi, h)$.

Note that points of $\mathcal{H}^{\text {in }}$ can equally well be thought of as equivalence classes of triples $\left(\boldsymbol{a}, a_{0}, f\right)$, where $\boldsymbol{a}=\left\{a_{1}, \ldots, a_{r}\right\} \in \mathcal{U}_{r}, a_{0} \in \mathcal{P}^{1} \backslash \boldsymbol{a}$ and $f: \Gamma=\pi_{1}\left(\mathcal{P}^{1} \backslash \boldsymbol{a}, a_{0}\right) \rightarrow G$ is a surjection that does not factor through the canonical map $\Gamma \rightarrow \Gamma_{i}$, for any $i$. Two such triples $\left(\boldsymbol{a}, a_{0}, f\right)$ and $\left(\tilde{\boldsymbol{a}}, \tilde{a}_{0}, \tilde{f}\right)$ are called equivalent if $\boldsymbol{a}=\tilde{\boldsymbol{a}}$ and there is a path $\gamma$ from $a_{0}$ to $\tilde{a}_{0}$ in $\mathcal{P}^{1} \backslash \boldsymbol{a}$ such that the induced map $\gamma^{*}: \pi_{1}\left(\mathcal{P}^{1} \backslash \boldsymbol{a}, a_{0}\right) \rightarrow \pi_{1}\left(\mathcal{P}^{1} \backslash \boldsymbol{a}, \tilde{a}_{0}\right)$ satisfies $\tilde{f} \circ \gamma^{*}=f$.

Here is the correspondence between the above pairs and triples. Let $\chi: \hat{X} \rightarrow \mathcal{P}^{1}$ be a Galois cover with $r$ branch points $a_{1}, \ldots, a_{r}$, let $\boldsymbol{a}=\left\{a_{1}, \ldots, a_{r}\right\}$ and $a_{0} \in \mathcal{P}^{1} \backslash \boldsymbol{a}$. Set $\Gamma=\pi_{1}\left(\mathcal{P}^{1} \backslash \boldsymbol{a}, a_{0}\right)$ as above. Depending on the choice of a base point $\hat{x} \in \chi^{-1}\left(a_{0}\right)$, we get a surjection $\iota: \Gamma \rightarrow \operatorname{Aut}\left(\hat{X} / \mathcal{P}^{1}\right)$ as follows: For each path $\gamma$ representing an element of $\Gamma$, let $\hat{y}$ be the endpoint of the unique lift of $\gamma$ to $\hat{X} \backslash \chi^{-1}(\boldsymbol{a})$ with initial point $\hat{x}$; then $\iota$ sends $\gamma$ to the unique element $\epsilon$ of $\operatorname{Aut}\left(\hat{X} / \mathcal{P}^{1}\right)$ with $\epsilon(\hat{x})=\hat{y}$. Now $h$ and $f$ are related by $f=h \circ \iota$, and $U_{\chi}=\operatorname{ker}(f)(=\operatorname{ker}(\iota))$. Varying $\hat{x}$ over $\chi^{-1}\left(a_{0}\right)$ means composing $\iota$ with inner automorphisms of Aut $\left(\hat{X} / \mathcal{P}^{1}\right)$. Therefore $h$ and $f$ determine each other up to inner automorphisms of $G$, which is compatible with the equivalence of pairs (resp., triples).

We have a natural map $\Lambda: \mathcal{H}^{\text {in }} \rightarrow \mathcal{H}^{\text {ab }}$ sending $|\chi, h|$ to the class of the cover $\varphi: \hat{X} / h^{-1}(U) \rightarrow \mathcal{P}^{1}$ induced by $\chi: \hat{X} \rightarrow \mathcal{P}^{1}$. Then, in the notation of the preceding paragraph, $f^{-1}(U)$ is one of the subgroups of $\Gamma$ corresponding to $\varphi$. Since $U$ does not contain a non-trivial normal subgroup of $G$, the intersection of all conjugates of $f^{-1}(U)$ equals the kernel of $f$. It follows that $\varphi$ has exactly the same $r$ branch points as does $\chi$ (namely, if $\operatorname{ker}\left(\Gamma \rightarrow \Gamma_{i}\right.$ ) would lie in $f^{-1}(U)$, then it would also be in $\operatorname{ker}(f)$, a contradiction). Thus $|\varphi|$ lies actually in $\mathcal{H}^{\text {ab }}=\mathcal{H}_{r}^{\text {ab }}(G, U)$, and $\Lambda$ is well defined. Let $\Psi: \mathcal{H}^{\text {ab }} \rightarrow \mathcal{U}_{r}$ and $\Psi^{\prime}: \mathcal{H}^{\text {in }} \rightarrow \mathcal{U}_{r}$ be the maps sending $|\varphi|$ and $|\chi, h|$ to the set of branch points of $\varphi$ and $\chi$, respectively. Then $\Psi^{\prime}=\Lambda \circ \Psi$.

The sets $\mathcal{H}^{\text {ab }}$ and $\mathcal{H}^{\text {in }}$ carry a natural topology such that $\Psi, \Psi^{\prime}$ and $\Lambda$ become (unramified) coverings. For $\mathcal{H}^{\text {ab }}$ this is classical, going back to Hurwitz (cf. [Fu, 1.3]): To specify a neighborhood $\mathcal{N}=$ $\mathcal{N}\left(\boldsymbol{p} ; D_{1}, \ldots, D_{r}\right)$ of $\boldsymbol{p}=|\varphi|$ in $\mathcal{H}^{\text {ab }}$, choose pairwise disjoint discs $D_{1}, \ldots, D_{r}$ around the branch points $a_{1}, \ldots, a_{r}$ of $\varphi$. Then $\mathcal{N}$ consists of all $|\tilde{\varphi}|$ such that $\tilde{\varphi}$ has exactly one branch point in each $D_{i}$, and the two covers of $\mathcal{P}^{1} \backslash\left(D_{1} \cup \cdots \cup D_{r}\right)$ induced by $\varphi$ and $\tilde{\varphi}$ are equivalent. These $\mathcal{N}$ form a basis for the neighborhoods of $\boldsymbol{p}$ in $\mathcal{H}^{\text {ab }}$.

The analogous topology on $\mathcal{H}^{\text {in }}$ is defined as follows: To specify a neighborhood $\mathcal{N}^{\prime}$ of the point of $\mathcal{H}^{\text {in }}$ represented by the triple $\left(\boldsymbol{a}, a_{0}, f\right)$, choose $\operatorname{discs} D_{i}$ around $a_{i}$ as above, with $a_{0} \notin D_{1} \cup \cdots \cup D_{r}$. Then $\mathcal{N}^{\prime}$ consists of all points represented by the triples $\left(\tilde{\boldsymbol{a}}, a_{0}, \tilde{f}\right)$ such that $\tilde{\boldsymbol{a}}$ has exactly one point in each $D_{i}$, and $\tilde{f}$ is the composition of the canonical isomorphisms $\pi_{1}\left(\mathcal{P}^{1} \backslash \tilde{\boldsymbol{a}}, a_{0}\right) \cong \pi_{1}\left(\mathcal{P}^{1} \backslash\left(D_{1} \cup \cdots \cup D_{r}\right), a_{0}\right) \cong \pi_{1}\left(\mathcal{P}^{1} \backslash \boldsymbol{a}, a_{0}\right)$ with $f$. Again these $\mathcal{N}^{\prime}$ form a basis for the topology. manifold.

Through the coverings $\Psi$ and $\Psi^{\prime}$, the spaces $\mathcal{H}^{\text {ab }}$ and $\mathcal{H}^{\text {in }}$ naturally inherit a structure of complex

$\S 1.3$. Monodromy action on the fibers of $\mathcal{H}^{\text {ab }}$ and $\mathcal{H}^{\text {in }}$ : To determine the equivalence class of the covering $\Psi: \mathcal{H}^{\mathrm{ab}} \rightarrow \mathcal{U}_{r}$ one needs to identify the natural permutation representation of $H_{r}=\pi_{1}\left(\mathcal{U}_{r}, \boldsymbol{b}\right)$ on the fiber $\Psi^{-1}(\boldsymbol{b})$. Recall that this action is defined as follows: The element of $H_{r}$ represented by a closed path $\omega$ sends a point $\boldsymbol{p} \in \psi^{-1}(\boldsymbol{b})$ to the endpoint of the unique lift of $\omega$ with initial point $\boldsymbol{p}$. Similarly for $\Psi^{\prime}$. 
This depends on the choice of generators $\gamma_{1}, \ldots, \gamma_{r}$ for the fundamental group $\Gamma_{0}=\pi_{1}\left(\mathcal{P}^{1} \backslash \boldsymbol{b}, b_{0}\right)$, where $b_{0}$ is a base point in $\mathcal{P}^{1} \backslash \boldsymbol{b}$ that we fix now once and for all; we choose $b_{0} \neq \infty$ such that no line through $b_{0}$ contains more than one of $b_{1}, \ldots, b_{r}$. Choose a disc $D$ centered at $b_{0}$ such that all the $b_{i}$ 's are contained in the interior of $D$. Partition $D$ into sectors $S_{1}, \ldots, S_{r}$ such that $b_{i}$ is in the interior of $S_{i}$ for $i=1, \ldots, r$. The numbering of the $b_{i}$ 's should be chosen so that the sectors $S_{1}, \ldots, S_{r}$ appear in this order in clockwise direction around the disc. Let $\gamma_{i}$ be the path with initial and end-point $b_{0}$, travelling clockwise along the boundary of $S_{i}$. By abuse, we don't distinguish between the paths $\gamma_{i}$ and their homotopy classes in $\Gamma_{0}$. Then $\Gamma_{0}$ is a free group on generators $\gamma_{1}, \ldots, \gamma_{r-1}$, and $\gamma_{1} \cdots \gamma_{r}=1$. (The latter relation can be seen especially clearly in the way we chose the $\gamma_{i}$ 's, since the product $\gamma_{1} \cdots \gamma_{r}$ is equal in $\Gamma_{0}$ to a path going from $b_{0}$ on a straight line to the boundary of $D$, travelling once around this bounding circle, and then returning to $b_{0}$; this path is clearly trivial in $\Gamma_{0}$ ).

Generating Paths: Paths made from sectors.

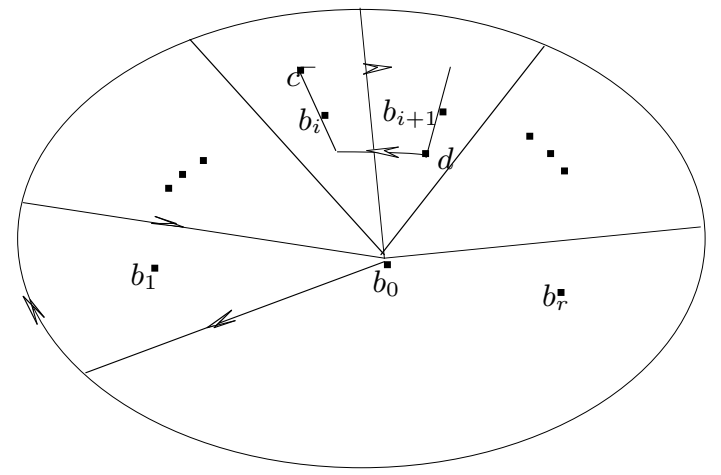

Let $N$ be the normal subgroup of $\Gamma_{0}$ generated by the conjugates of $\gamma_{r}$. Then $\Gamma_{0} / N$ is generated by $\gamma_{1} N, \ldots, \gamma_{r-2} N$, and the images of these elements under the natural map $\Gamma_{0} / N \rightarrow \pi_{1}\left(\mathcal{P}^{1} \backslash\left\{b_{1}, \ldots, b_{r-1}\right\}, b_{0}\right)$ are free generators of the latter group. Hence this map is an isomorphism. With $r$ replaced by any $i=1, \ldots, r$, we conclude that the kernel of the natural map $\Gamma_{0} \rightarrow \pi_{1}\left(\left(\mathcal{P}^{1} \backslash \boldsymbol{b}\right) \cup\left\{b_{i}\right\}, b_{0}\right)$ equals the normal subgroup of $\Gamma_{0}$ generated by the conjugates of $\gamma_{i}$. This implies the following: Assume $\varphi^{\prime}: X^{\prime} \rightarrow \mathcal{P}^{1}$ is a cover with branch points among $b_{1}, \ldots, b_{r}$, and one of the subgroups of $\Gamma_{0}$ corresponding to $\varphi^{\prime}$ is of the form $f^{-1}(U)$, where $f: \Gamma_{0} \rightarrow G$ is a surjection. Then $\varphi^{\prime}$ has exactly $r$ branch points if and only if $f\left(\gamma_{i}\right) \neq 1$ for all $i=1, \ldots, r$ (cf. $\S 1.2)$.

If now $\varphi$ is a cover of $\mathcal{P}^{1}$ with $|\varphi| \in \psi^{-1}(\boldsymbol{b})$, then by definition there is a surjection $f: \Gamma_{0} \rightarrow G$ with $f^{-1}(U)$ conjugate to $U_{\varphi}$. The kernel of $f$ equals the intersection of all subgroups in the class $\left[U_{\varphi}\right]$, hence it depends only on $|\varphi|$. Thus $f$ is determined by $|\varphi|$ up to composition with elements of $\operatorname{Aut}(G, U)$. Conversely, $|\varphi|$ is determined by $f$ since $\left[U_{\varphi}\right]=f^{-1}([U])$. Also, each surjection $f: \Gamma_{0} \rightarrow G$ with $f\left(\gamma_{i}\right) \neq 1$ for all $i=1, \ldots, r$ gives rise to some $|\varphi| \in \Psi^{-1}(\boldsymbol{b})$ (by the preceding paragraph). Furthermore, $f$ is determined by the $r$-tuple $\left(\sigma_{1}, \ldots, \sigma_{r}\right)=\left(f\left(\gamma_{1}\right), \ldots, f\left(\gamma_{r}\right)\right) \in \mathcal{E}_{r}(G)$ (see $\S 1.1$ for the definition of $\left.\mathcal{E}_{r}(G)\right)$. Since $\Gamma_{0}$ is free on $\gamma_{1}, \ldots, \gamma_{r-1}$, each $\left(\sigma_{1}, \ldots, \sigma_{r}\right) \in \mathcal{E}_{r}=\mathcal{E}_{r}(G)$ occurs in this way. Thus we get a bijection between the points $|\varphi|$ in the fiber $\Psi^{-1}(\boldsymbol{b})$ and the set $\mathcal{E}_{r}^{\text {ab }}\left(=\mathcal{E}_{r}\right.$ modulo $\left.\operatorname{Aut}(G, U)\right)$ of $\operatorname{Aut}(G, U)$-classes of the $r$-tuples $\left(\sigma_{1}, \ldots, \sigma_{r}\right)$. Via this bijection, we get an action of $H_{r}=\pi_{1}\left(\mathcal{U}_{r}, \boldsymbol{b}\right)$ on $\mathcal{E}_{r}^{\text {ab }}$.

Similarly, we get a bijection between the points $|\chi, h|$ in the fiber $\left(\Psi^{\prime}\right)^{-1}(\boldsymbol{b})$ and the set $\mathcal{E}_{r}^{\text {in }}\left(=\mathcal{E}_{r}\right.$ modulo $\operatorname{Inn}(G)$ ): Given $(\chi, h)$ define $f=h \circ \iota: \Gamma_{0} \rightarrow G$ as in $\S 1.2$, and associate with it the class of $\left(\sigma_{1}, \ldots, \sigma_{r}\right)=\left(f\left(\gamma_{1}\right), \ldots, f\left(\gamma_{r}\right)\right)$ in $\mathcal{E}_{r}^{\text {in }}$. This yields the desired bijection, since $f$ is determined by $|\chi, h|$ up to composition with inner automorphisms of $G$ (see $\S 1.2$ ). This bijection induces an action of $H_{r}$ on $\mathcal{E}_{r}^{\text {in }}$. 
We are going to describe explicitly the action of the generators $Q_{1}, \ldots, Q_{r-1}$ of $H_{r}$ (from $\S 1.1$ ) on the sets $\mathcal{E}_{r}^{\text {ab }}$ and $\mathcal{E}_{r}^{\text {in }}$. First we need an explicit description of $Q_{i}$ for $i=1, \ldots, r-1$ (c.f. [BF], [Fu, 1.4]): Choose a point $c$ on the line $\overline{b_{0} b_{i}}$ strictly between $b_{i}$ and the boundary of $D$, and closer to this boundary than $b_{i+1}$; further choose a point $d$ on the line $\overline{b_{i+1} b_{0}}$ strictly between $b_{i+1}$ and $b_{0}$, and closer to $b_{0}$ than $b_{i}$. We can represent $Q_{i}$ by a path $\boldsymbol{a}(t)=\left\{a_{1}(t), \ldots, a_{r}(t)\right\}$ in $\mathcal{U}_{r}, 0 \leq t \leq 1$, where $a_{j}(t)$ is the constant path $b_{j}$ for $j \neq i, i+1$; and $a_{i}(t)$ is a path going from $b_{i}$ on a straight line to $c$, then travelling on a circular arc around $b_{0}$ in clockwise direction until it meets the line $\overline{b_{0} b_{i+1}}$, from where it continues on this line to $b_{i+1}$; and $a_{i+1}(t)$ is a path going from $b_{i+1}$ on a straight line to $d$, then travelling on a circular arc around $b_{0}$ in counter-clockwise direction until it meets the line $b_{0} b_{i}$, from where it continues on this line to $b_{i}$.

Having fixed all the above data, the action of $Q_{i}$ on $\mathcal{E}_{r}^{\text {ab }}$ and on $\mathcal{E}_{r}^{\text {in }}$ is now given by the following rule: $Q_{i}$ sends the class of $\left(\sigma_{1}, \ldots, \sigma_{r}\right) \in \mathcal{E}_{r}$ to the class of

$$
\left(\sigma_{1}, \ldots, \sigma_{i-1}, \sigma_{i+1}, \sigma_{i+1}^{-1} \sigma_{i} \sigma_{i+1}, \sigma_{i+2}, \ldots, \sigma_{r}\right) .
$$

For the case of $\mathcal{H}^{\text {ab }}$, this observation goes back to Hurwitz [Hur] (see also [Fr,1] and [Fu, 1.4]). We give a proof in $\S 1.4$.

By the theory of covering spaces (and the above identifications), the connected components of $\mathcal{H}^{\text {ab }}$ and $\mathcal{H}^{\text {in }}$ now correspond to the orbits of $H_{r}$ on $\mathcal{E}_{r}^{\text {ab }}$ and $\mathcal{E}_{r}^{\text {in }}$, respectively. Note that for each $r$-tuple $\mathbf{C}$ of conjugacy classes of $G$, the subsets $\mathrm{Ni}(\mathbf{C})^{\text {ab }}$ and $\mathrm{Ni}(\mathbf{C})^{\text {in }}$ of $\mathcal{E}_{r}^{\text {ab }}$ and $\mathcal{E}_{r}^{\text {in }}$, respectively (see $\S 1.1$ ), are invariant under the action of $H_{r}$; hence these subsets are unions of $H_{r}$-orbits. Let $\mathcal{H}(\mathbf{C})^{\text {ab }}\left(\right.$ resp., $\left.\mathcal{H}(\mathbf{C})^{\text {in }}\right)$ denote the union of the corresponding connected components of $\mathcal{H}^{\text {ab }}$ (resp. $\left.\mathcal{H}^{\text {in }}\right)$. We call these subspaces $\mathcal{H}(\mathbf{C})^{\text {ab }}$ and $\mathcal{H}(\mathbf{C})^{\text {in }}$ Hurwitz spaces. Each component of $\mathcal{H}^{\text {ab }}$ (resp., $\mathcal{H}^{\text {in }}$ ) belongs to such a Hurwitz space.

It is a basic problem to decide when the Hurwitz spaces themselves are connected. That is, when $H_{r}$ acts transitively on $\mathrm{Ni}(\mathbf{C})^{\text {ab }}$ or $\mathrm{Ni}(\mathbf{C})^{\text {in }}$. For the case of simply branched covers (i.e., $\mathbf{C}=(\mathrm{C}, \ldots, \mathrm{C})$ where C is the class of transpositions in $G=S_{n}(n>2)$, and $\left.U=S_{n-1}\right)$ this was done by Clebsch [Cl]. In the Appendix we present a far more general result in this direction, due to Conway and Parker [CP].

$\S$ 1.4. The action of $Q_{i}$ on $\mathcal{E}_{r}^{\text {ab }}$ and $\mathcal{E}_{r}^{\text {in }}$ : Here we indicate how the action of $Q_{i}$ gives formula (2). We represent $Q_{i}$ by the path $\boldsymbol{a}(t)$ from $\S 1.3$. Choose another disc $D^{\prime}$ around $b_{0}$ that contains the point $d$ in its interior, and $b_{i}, b_{i+1}$ are not in $D^{\prime}$. (This is possible by the choice of $d$ ). Let $R_{i}$ be the interior of the sector of $D^{\prime}$ cut out by $S_{i} \cup S_{i+1}$, and let $T_{i}$ be the interior of $\left(S_{i} \cup S_{i+1}\right) \backslash D^{\prime}$. Let $S_{j}^{0}$ be the interior of $S_{j}$, and $P=\mathcal{P}^{1} \backslash \mathbf{b}$. We may assume that the paths $a_{i}(t)$ and $a_{i+1}(t)$ reach the boundary between $S_{i}$ and $S_{i+1}$ at the same time $t=1 / 2$, and that $a_{i+1}(t) \in R_{i}$ exactly for $1 / 4<t<3 / 4$. Note that the path $a_{i}(t)$ remains always in $T_{i}$. The inclusions between the respective spaces give canonical isomorphisms:

$$
\begin{aligned}
& \pi_{1}\left(\mathcal{P}^{1} \backslash \boldsymbol{a}(t), b_{0}\right) \cong \pi_{1}\left(P \backslash\left(S_{i}^{0} \cup S_{i+1}^{0}\right), b_{0}\right) \stackrel{\text { def }}{=} \Gamma^{(0)} \text { for } \mathrm{t}<1 / 2 ; \\
& \pi_{1}\left(\mathcal{P}^{1} \backslash \boldsymbol{a}(t), b_{0}\right) \cong \pi_{1}\left(P \backslash\left(R_{i} \cup T_{i}\right), b_{0}\right) \stackrel{\text { def }}{=} \Gamma^{(1)} \quad \text { for } 1 / 4<\mathrm{t}<3 / 4 ; \text { and } \\
& \pi_{1}\left(\mathcal{P}^{1} \backslash \boldsymbol{a}(t), b_{0}\right) \cong \pi_{1}\left(P \backslash\left(S_{i}^{0} \cup S_{i+1}^{0}\right), b_{0}\right)=\Gamma^{(0)} \text { for } \mathrm{t}>1 / 2 .
\end{aligned}
$$

From (3) and (4) we get an isomorphism $\alpha_{1}: \Gamma^{(0)} \rightarrow \Gamma^{(1)}$ for each choice of $t$ with $1 / 4<t<1 / 2$, and this isomorphism is the same for all these $t$. Similarly, from (4) and (5) we get an isomorphism $\alpha_{2}: \Gamma^{(1)} \rightarrow$ $\Gamma^{(0)}$, independent of the choice of $t$ with $1 / 2<t<3 / 4$. With paths and their respective homotopy classes identified, one checks easily that the automorphism $\alpha=\alpha_{2} \circ \alpha_{1}$ of $\Gamma^{(0)}$ is given by

$$
\alpha\left(\gamma_{i+1}\right)=\gamma_{i}, \quad \alpha\left(\gamma_{i}\right)=\gamma_{i} \gamma_{i+1} \gamma_{i}^{-1}, \quad \alpha\left(\gamma_{j}\right)=\gamma_{j} \text { for } j \neq i, i+1
$$

The groups $\Gamma^{(0)}$ and $\Gamma_{0}$ are canonically isomorphic, under an isomorphism which identifies the classes of $\gamma_{1}, \ldots, \gamma_{r}$ in $\Gamma^{(0)}$ and $\Gamma_{0}$, respectively. We let $\alpha$ also denote the automorphism of $\Gamma_{0}$ given by (6). 
Now let $\boldsymbol{p}(t)$ be a lift of the path $\boldsymbol{a}(t)$ to $\mathcal{H}^{\text {ab }}$. Then for each $t$, the point $\boldsymbol{p}(t)$ is an equivalence class of covers of $\mathcal{P}^{1}$ with $\boldsymbol{a}(t)$ as its set of branch points. Thus $\boldsymbol{p}(t)$ corresponds to a conjugacy class $\Delta_{t}$ of subgroups of $\pi_{1}\left(\mathcal{P}^{1} \backslash \boldsymbol{a}(t), b_{0}\right)$; the image of $\Delta_{t}$ under the isomorphisms (3), (4) and (5) is independent of $t$ (under the respective conditions on $t$ ), by the definition of the topology on $\mathcal{H}^{\text {ab }}$. Thus the conjugacy classes $\Delta_{0}$ and $\Delta_{1}$ of subgroups of $\Gamma_{0}$ corresponding to the initial point $\boldsymbol{p}(0)$ and the endpoint $\boldsymbol{p}(1)$ of the path $\boldsymbol{p}(t)$, respectively, are related by $\Delta_{1}=\alpha\left(\Delta_{0}\right)$.

Finally, if $f_{0}: \Gamma_{0} \rightarrow G$ is a surjection with $f_{0}^{-1}(U) \in \Delta_{0}$, then $f_{1} \stackrel{\text { def }}{=} f_{0} \circ \alpha^{-1}$ is a surjection $\Gamma_{0} \rightarrow G$ with

$$
f_{1}^{-1}(U)=\alpha \circ f_{0}^{-1}(U) \in \alpha\left(\Delta_{0}\right)=\Delta_{1} .
$$

Thus $\left(\sigma_{1}, \ldots, \sigma_{r}\right)=\left(f_{0}\left(\gamma_{1}\right), \ldots, f_{0}\left(\gamma_{r}\right)\right)$ is an $r$-tuple representing the element of $\mathcal{E}_{r}^{\text {ab }}$ corresponding to $\boldsymbol{p}(0)$, and $\left(\sigma_{1}, \ldots, \sigma_{i+1}, \sigma_{i+1}^{-1} \sigma_{i} \sigma_{i+1}, \ldots, \sigma_{r}\right)=\left(f_{1}\left(\gamma_{1}\right), \ldots, f_{1}\left(\gamma_{r}\right)\right)$ represents the element of $\mathcal{E}_{r}^{\text {ab }}$ corresponding to $\boldsymbol{p}(1)$. This proves that the action of $Q_{i}$ on $\mathcal{E}_{r}^{\text {ab }}$ is given by formula (2).

Now let $\boldsymbol{p}^{\prime}(t)$ be a lift of the path $\boldsymbol{a}(t)$ to $\mathcal{H}^{\text {in }}$. For each $t$, the point $\boldsymbol{p}^{\prime}(t)$ is represented by a triple $\left(\boldsymbol{a}(t), b_{0}, f_{t}\right)$ where $f_{t}: \pi_{1}\left(\mathcal{P}^{1} \backslash \boldsymbol{a}(t), b_{0}\right) \rightarrow G$ is a surjection. The surjections $\Gamma^{(0)} \rightarrow G$ (resp., $\left.\Gamma^{(1)} \rightarrow G\right)$ that are the composition of the isomorphisms (3) and (5) (resp., (4)) with $f_{t}$ are independent of $t$ (under the respective conditions on $t$ ), by the definition of the topology on $\mathcal{H}^{\text {in }}$. It follows that the map $f_{0}$ corresponding to $\boldsymbol{p}^{\prime}(0)$ and the map $f_{1}$ corresponding to $\boldsymbol{p}^{\prime}(1)$ are related by $f_{1}=f_{0} \circ \alpha^{-1}$. From this we conclude - as in the preceding paragraph - that $Q_{i}$ acts on $\mathcal{E}_{r}^{\text {in }}$ via formula $(2)$.

$\S 1.5$. The field of definition of a cover : For each cover $\varphi: X \rightarrow \mathcal{P}^{1}$ (of connected compact Riemann surfaces) the space $X$ has a unique structure as algebraic variety defined over $\mathcal{C}$ (compatible with its analytic structure) such that $\varphi$ becomes an algebraic morphism. This is Riemann's existence theorem. We say that $\varphi$ can be defined over some subfield $k$ of $\mathcal{C}$ if $X$ can be given a structure of variety defined over $k$ such that $\varphi$ becomes a morphism defined over $k$.

Let $\boldsymbol{a}=\left\{a_{1}, \ldots, a_{r}\right\} \in \mathcal{U}_{r}$ be the set of branch points of $\varphi$, and $k_{0}=\mathcal{Q}(\boldsymbol{a})$; thus $k_{0}$ is the field generated by the coefficients of the polynomial $\prod\left(x-a_{i}\right)$, where the product is over those $i=1, \ldots, r$ with $a_{i} \neq \infty$. Now let $k$ be any subfield of $\mathcal{C}$ over which $\varphi$ can be defined. Then the branch points $a_{1}, \ldots, a_{r}$ are algebraic over $k$, and the absolute Galois group $G_{k}$ permutes $a_{1}, \ldots, a_{r}$. Therefore $k_{0}$ is contained in $k$.

Conversely, $\varphi$ can be defined over the algebraic closure $F$ of $k_{0}$ in $\mathcal{C}$, in a unique way [Fr,1; Lemma 1.2]. Thus for each $\beta \in G_{k_{0}}$, we can form the cover $\varphi^{\beta}: X^{\beta} \rightarrow \mathcal{P}^{1}$ obtained from $\varphi: X \rightarrow \mathcal{P}^{1}$ through base change with $\beta$. Let $k_{1}$ be the fixed field in $F$ of the group of those $\beta$ for which $\varphi^{\beta}$ is equivalent to $\varphi$. We show that $k_{1}$ is contained in each subfield $k$ of $\mathcal{C}$ over which $\varphi$ can be defined: By the above $k_{0} \subset k$. If $c \in \mathcal{C}$ is algebraic over $k_{0}$ and not in $k$, then there exists $\beta^{\prime} \in G_{k}$ with $\beta^{\prime}(c) \neq c$. Since $\varphi$ can be defined over $k$ we have $\varphi^{\beta^{\prime}}$ equivalent to $\varphi$. Therefore the restriction of $\beta^{\prime}$ to an element of $G_{k_{0}}$ must fix $k_{1}$ elementwise, and thus $c \notin k_{1}$. This proves $k_{1} \subset k$.

Conversely $\varphi$ can actually be defined over $k_{1}$ if we have $\operatorname{Aut}\left(X / \mathcal{P}^{1}\right)=1$ [Fr,1; Theorem 5.1]. For completeness we give the argument here. The condition that $\operatorname{Aut}\left(X / \mathcal{P}^{1}\right)$ is trivial implies that for each $\beta \in G_{k_{1}}$ the isomorphism $\delta_{\beta}: X^{\beta} \rightarrow X$ with $\varphi \circ \delta_{\beta}=\varphi^{\beta}$ is unique. This forces the maps $\delta_{\beta}$ to satisfy Weil's cocycle condition. By Weil's criterion [W], $X$ can be defined over $k_{1}$ such that $\delta_{\beta}: X=X^{\beta} \rightarrow X$ is the identity for each $\beta \in G_{k_{1}}$. Then also $\varphi: X \rightarrow \mathcal{P}^{1}$ is defined over $k_{1}$.

Also if $\varphi$ is a Galois cover then it can be defined over $k_{1}$ (but perhaps not together with its automorphisms); this was noted in [CHa]. (The proof is similar as above: Fix a point $z \in X$ lying over a $k_{1}$-point of $\mathcal{P}^{1}$ that is not a branch point, and normalize the $\delta_{\beta}$ such that $\delta_{\beta}\left(z^{\beta}\right)=z$ ). Thus if either $\varphi$ is a Galois cover or $\operatorname{Aut}\left(X / \mathcal{P}^{1}\right)=1$, then $\varphi$ has a unique minimal field of definition $k_{1} \subset \mathcal{C}$. 


\section{THE MAIN THEOREM AND SOME CONSEQUENCES}

$\S$ 2.1. The Main Theorem: Define a point $\boldsymbol{p}=|\varphi|$ of $\mathcal{H}^{\text {ab }}$ to be a $\overline{\mathcal{Q}}$-point if the branch points of $\varphi$ are algebraic over $\mathcal{Q}$. Then $\varphi$ can be defined over $\overline{\mathcal{Q}}$ by $\S 1.5$. The notion of a $\overline{\mathcal{Q}}$-point of $\mathcal{H}^{\text {in }}$ is defined similarly. There is a natural action of the absolute Galois group $G_{\mathcal{Q}}$ on the $\overline{\mathcal{Q}}$-points of $\mathcal{H}^{\text {ab }}$ : The element $\beta \in G_{\mathcal{Q}}$ sends $|\varphi|$ to $\left|\varphi^{\beta}\right|$, where $\varphi^{\beta}: X^{\beta} \rightarrow \mathcal{P}^{1}$ is the cover obtained from $\varphi: X \rightarrow \mathcal{P}^{1}$ through base change with $\beta$. Note that if $|\varphi| \in \mathcal{H}^{\mathrm{ab}}=\mathcal{H}_{r}^{\mathrm{ab}}(G, U)$ then also $\left|\varphi^{\beta}\right| \in \mathcal{H}_{r}^{\mathrm{ab}}(G, U)$, because the pair $(G, U)$ can be recovered from $\varphi$ as the pair $\left(\operatorname{Aut}\left(\hat{X} / \mathcal{P}^{1}\right)\right.$, Aut $(\hat{X} / X)$ ), where $\hat{X} \rightarrow X \stackrel{\varphi}{\longrightarrow} \mathcal{P}^{1}$ is a Galois closure of $\varphi$ (i.e., $\hat{X} \rightarrow \mathcal{P}^{1}$ is a minimal Galois cover factoring through $\varphi$ ).

Similarly, we get an action of $G_{\mathcal{Q}}$ on the $\overline{\mathcal{Q}}$-points of $\mathcal{H}^{\text {in }}$ : The element $\beta \in G_{\mathcal{Q}}$ sends the point $|\chi, h|$ to $\left|\chi^{\beta}, h \circ \beta^{-1}\right|$, where $h \circ \beta^{-1}: \operatorname{Aut}\left(\hat{X}^{\beta} / \mathcal{P}^{1}\right) \rightarrow G$ is the isomorphism sending $A^{\beta}$ to $h(A)$ for every $A \in \operatorname{Aut}\left(\hat{X} / \mathcal{P}^{1}\right)$. So it is natural to expect that $\mathcal{H}^{\text {ab }}$ and $\mathcal{H}^{\text {in }}$ have a structure as varieties defined over $\mathcal{Q}$ such that the resulting action of $G_{\mathcal{Q}}$ on the $\overline{\mathcal{Q}}$-points is as above. Such a $\mathcal{Q}$ - structure on $\mathcal{H}^{\text {ab }}$ and $\mathcal{H}^{\text {in }}$ is then necessarily unique. Its existence is our main theorem, which will be proved in $\S 3-5$ :

Theorem 1: Let $G$ be a finite group, and $U \leq G$ a proper subgroup that does not contain a non-trivial normal subgroup of $G$. Let $r \geq 3$ be an integer such that $G$ can be generated by $r-1$ elements. Then the spaces $\mathcal{H}^{\mathrm{ab}}=\mathcal{H}_{r}^{\mathrm{ab}}(G, U)$ and $\mathcal{H}^{\text {in }}=\mathcal{H}_{r}^{\text {in }}(G)$ have a unique structure as (reducible) algebraic varieties defined over $\mathcal{Q}$ (compatible with their analytic structure) so that the maps

$$
\mathcal{H}^{\text {in }} \stackrel{\Lambda}{\longrightarrow} \mathcal{H}^{\mathrm{ab}} \stackrel{\Psi}{\longrightarrow} \mathcal{U}_{r}
$$

are algebraic morphisms defined over $\mathcal{Q}$, and the following hold:

(a) If $\boldsymbol{p}=|\varphi|$ is a point of $\mathcal{H}^{\mathrm{ab}}$ such that the branch points of $\varphi$ are algebraic over some subfield $k$ of $\mathcal{C}$, then $\boldsymbol{p}$ is algebraic over $k$, and each automorphism $\beta$ of $\bar{k}$ sends $\boldsymbol{p}=|\varphi|$ to $\left|\varphi^{\beta}\right|$.

(b) If $\boldsymbol{p}^{\prime}=|\chi, h|$ is a point of $\mathcal{H}^{\text {in }}$ such that the branch points of $\chi$ are algebraic over some subfield $k$ of $\mathcal{C}$, then $\boldsymbol{p}^{\prime}$ is algebraic over $k$, and each automorphism $\beta$ of $\bar{k}$ sends $\boldsymbol{p}^{\prime}=|\chi, h|$ to $\left|\chi^{\beta}, h \circ \beta^{-1}\right|$ (where $h \circ \beta^{-1}$ is to be understood as in the preceding paragraph). 
Furthermore, the absolutely irreducible components of $\mathcal{H}^{\text {ab }}$ and $\mathcal{H}^{\text {in }}$ (defined over $\overline{\mathcal{Q}}$ ) coincide with the connected components (in the topology from §1.2). Let $\mathbf{C}$ be an $r$-tuple of conjugacy classes of $G$ such that $\mathrm{Ni}(\mathbf{C})^{\text {in }}$ is non-empty. Then $\mathbf{C}$ is rational (as defined in $\S 1.1$ ) if and only if the subspace $\mathcal{H}(\mathbf{C})^{\text {in }}$ of $\mathcal{H}^{\text {in }}$ is defined over $\mathcal{Q}$. In addition, $\mathcal{H}(\mathbf{C})^{\mathrm{in}}$ is absolutely irreducible if and only if the Hurwitz monodromy group $H_{r}$ acts transitively on $\mathrm{Ni}(\mathbf{C})^{\text {in }}$.

We remark that the subspace $\mathcal{H}(\mathbf{C})^{\text {ab }}$ of $\mathcal{H}^{\text {ab }}$ may be defined over $\mathcal{Q}$ even for non-rational C. But if $\mathbf{C}$ is rational then $\mathcal{H}(\mathbf{C})^{\mathrm{ab}}$ is certainly defined over $\mathcal{Q}$. Also, $\mathcal{H}(\mathbf{C})^{\mathrm{ab}}$ is absolutely irreducible if and only if $H_{r}$ is transitive on $\mathrm{Ni}(\mathbf{C})^{\text {ab }}$. Here is the significance of Theorem 1 for the Inverse Galois Problem:

Corollary 1: Keep the hypotheses of Theorem 1, and let $K$ be any field of characteristic 0 . Assume additionally that the group $G$ has trivial center. Then $G$ is regular over $K$ with $r$ branch points if and only if $\mathcal{H}^{\text {in }}=\mathcal{H}_{r}^{\text {in }}(G)$ has a $K$-rational point. More precisely:

(a) For each point $\boldsymbol{p}=|\varphi|$ of $\mathcal{H}^{\mathrm{ab}}$ the field $\mathcal{Q}(\boldsymbol{p})$ is the minimal field of definition of the cover $\varphi$, if either $U=1$ or $U$ is self-normalizing in $G$ (cf. $\S 1.5$ ).

(b) For each point $\boldsymbol{p}^{\prime}=|\chi, h|$ of $\mathcal{H}^{\text {in }}$ the field $\mathcal{Q}\left(\boldsymbol{p}^{\prime}\right)$ is the minimal subfield $k^{\prime}$ of $\mathcal{C}$ such that the cover $\chi: \hat{X} \rightarrow \mathcal{P}^{1}$ together with all its automorphisms can be defined over $k^{\prime}$. The resulting function field extension $L / k^{\prime}(x)$, where $L=k^{\prime}(\hat{X})$, is Galois with Galois group isomorphic to $G$, and $L$ is regular over $k^{\prime}$.

Proof of Corollary 1: (a) Set $k_{0}=\mathcal{Q}(\Psi(\boldsymbol{p}))$ as in $\S 1.5$. Then $\boldsymbol{p}$ is algebraic over $k_{0}$, and clearly $k=\mathcal{Q}(\boldsymbol{p})$ is the fixed field of the group of all $\beta \in G_{k_{0}}$ with $\boldsymbol{p}^{\beta}=\boldsymbol{p}$. By Theorem $1, k$ is the fixed field of the group of all $\beta \in G_{k_{0}}$ with $\varphi^{\beta}$ equivalent to $\varphi$. From $\S 1.5$, this proves (a) (in view of (1)).

(b) Set $\left.k_{0}=\mathcal{Q}\left(\Psi^{\prime}\left(\boldsymbol{p}^{\prime}\right)\right)\right)$. If $k^{\prime}$ is a field such that $\chi$ together with all its automorphisms is defined over $k^{\prime}$, then $\left(\chi^{\beta}, h \circ \beta^{-1}\right)=(\chi, h)$ for all $\beta \in G_{k^{\prime}}$, and $k_{0} \subset k^{\prime}$. By Theorem $1, G_{k^{\prime}}$ fixes $\boldsymbol{p}^{\prime}$ and so $\mathcal{Q}\left(\boldsymbol{p}^{\prime}\right) \subset k^{\prime}$.

It remains to show that $\chi$ together with all its automorphisms can actually be defined over $K^{\prime}=\mathcal{Q}\left(\boldsymbol{p}^{\prime}\right)$. As in $\S 1.5$, the cover $\chi$ can be defined over $\bar{k}_{0}=\bar{K}^{\prime}$. For all $\beta \in G_{K^{\prime}}$, we have $\left(\chi^{\beta}, h \circ \beta^{-1}\right)$ equivalent to $(\chi, h)$ by Theorem 1. Thus there exist isomorphisms $\delta_{\beta}: \hat{X}^{\beta} \rightarrow \hat{X}$ over $\mathcal{P}^{1}$ with $h \circ \beta^{-1}\left(\delta_{\beta}^{-1} A \delta_{\beta}\right)=h(A)$ for each $A \in \operatorname{Aut}\left(\hat{X} / \mathcal{P}^{1}\right)$. The latter implies that $A^{\beta}=\delta_{\beta}^{-1} A \delta_{\beta}$. In particular, since $G$ has trivial center, $\delta_{\beta}$ is uniquely determined by $\beta$. This uniqueness again forces the $\delta_{\beta}$ to satisfy Weil's cocycle condition, and by Weil's criterion it follows that the cover $\chi: \hat{X} \rightarrow \mathcal{P}^{1}$ can be defined over $K^{\prime}$ so that $\delta_{\beta}: \hat{X}=\hat{X}^{\beta} \rightarrow \hat{X}$ becomes the identity. Then $A^{\beta}=\delta_{\beta}^{-1} A \delta_{\beta}=A$ for all $A \in \operatorname{Aut}\left(\hat{X} / \mathcal{P}^{1}\right)$. That is, all automorphisms of $\chi$ are defined over $K^{\prime}$. This proves the first claim in (b). The second follows immediately.

For the first assertion in Corollary 1 , note that if $\mathcal{H}^{\text {in }}$ has a point $\boldsymbol{p}^{\prime}$ over the field $K$ then we may assume that $\boldsymbol{p}^{\prime}$ is a complex point (because $k^{\prime}=\mathcal{Q}\left(\boldsymbol{p}^{\prime}\right)$ is finitely generated over $\mathcal{Q}$ ). Then (b) yields a regular Galois extension $L / k^{\prime}(x)$ with group $G$ and with $r$ branch points. Tensoring with $K$ shows that $G$ is regular over $K$ with $r$ branch points. Conversely, suppose the latter holds. Then there is a finitely generated subfield $K^{\prime}$ of $K$ such that $G$ is regular over $K^{\prime}$ with $r$ branch points; we may assume that $K^{\prime}$ is a subfield of $\mathcal{C}$. Hence there is a Galois cover $\chi: \hat{X} \rightarrow \mathcal{P}^{1}$ with $r$ branch points and an isomorphism $h: \operatorname{Aut}\left(\hat{X} / \mathcal{P}^{1}\right) \rightarrow G$ such that $\chi$ together with all its automorphisms is defined over $K^{\prime}$. By (b) it follows that $\mathcal{Q}\left(\boldsymbol{p}^{\prime}\right) \subset K^{\prime}$, where $\boldsymbol{p}^{\prime}=|\chi, h| \in \mathcal{H}^{\text {in }}$. Thus $\boldsymbol{p}^{\prime}$ is a point of $\mathcal{H}^{\text {in }}$ that is rational over $K$.

Corollary 1 shows that the $\mathcal{Q}$-structure on $\mathcal{H}^{\text {in }}$ (and on its Hurwitz subspaces) is crucial for the Inverse Galois Problem. See [Fr,3] for a discussion in the case $r \leq 4$, including a relation between Hurwitz spaces and modular curves for $r=4$. In the following example we determine the $\mathcal{Q}$-structure of Hurwitz spaces $\mathcal{H}(\mathbf{C})^{\text {in }}$ for which $\mathbf{C}$ satisfies the rigidity condition of Thompson [Th]. 
Example: Hurwitz spaces and rigidity. Assume $G$ has trivial center. The $r$-tuple $\mathbf{C}=\left(C_{1}, \ldots, C_{r}\right)$ of conjugacy classes of $G$ is called rigid if the tuples $\left(\sigma_{1}, \ldots, \sigma_{r}\right) \in \mathcal{E}_{r}(G)$ with $\sigma_{i} \in C_{i}$ form a single (nonempty) orbit under $\operatorname{Inn}(G)$. Assume this holds. For simplicity we consider only the case that the $C_{i}$ 's are all distinct. (The transition to the general case is immediate). Then the elements of $\mathrm{Ni}(\mathbf{C})^{\mathrm{in}}$ correspond to the permutations of $C_{1}, \ldots, C_{r}$, and $Q_{i}$ acts (via formula (2)) as the transposition $(i, i+1)$ on these permutations (for $i=1, \ldots, r-1$ ). Thus the Hurwitz group $H_{r}$ acts through its natural $S_{r}$-quotient on $\mathrm{Ni}(\mathbf{C})^{\text {in }}$, more precisely, through the regular permutation representation of $S_{r}$. This determines the permutation representation of $H_{r}$ that defines the equivalence class of the cover $\mathcal{H}(\mathbf{C})^{\text {in }} \rightarrow \mathcal{U}_{r}(\mathrm{cf}$. $\S 1.3)$. It follows that this cover is equivalent over $\overline{\mathcal{Q}}$ to the cover

$$
\mathcal{U}^{(r)} \stackrel{\text { def }}{=}\left\{\left(x_{1}, \ldots, x_{r}\right) \in\left(\mathcal{P}^{1}\right)^{r}: x_{i} \neq x_{j} \text { for } i \neq j\right\} \rightarrow \mathcal{U}_{r}
$$

where the (ordered) tuple $\left(x_{1}, \ldots, x_{r}\right)$ is mapped to the set $\left\{x_{1}, \ldots, x_{r}\right\} \in \mathcal{U}_{r}$.

Fix a $\overline{\mathcal{Q}}$-isomorphism $\theta: \mathcal{H}(\mathbf{C})^{\text {in }} \rightarrow \mathcal{U}^{(r)}$ over $\mathcal{U}_{r}$. Now assume additionally that the $r$-tuple $\mathbf{C}$ is rational. Then $\mathcal{H}(\mathbf{C})^{\text {in }}$ is an absolutely irreducible variety defined over $\mathcal{Q}$ (by Theorem 1 ). For $\beta \in G_{\mathcal{Q}}$, consider the map $\theta^{\beta}: \mathcal{H}(\mathbf{C})^{\text {in }} \rightarrow \mathcal{U}^{(r)}$ obtained by base change with $\beta$ (where $\mathcal{U}^{(r)}$ is viewed as $\mathcal{Q}$-variety in the natural way). Set $c(\beta)=\theta^{-1} \theta^{\beta}$, an element of $\operatorname{Aut}\left(\mathcal{H}(\mathbf{C})^{\text {in }} / \mathcal{U}_{r}\right)$.

Consider a point $\boldsymbol{p} \in \mathcal{H}(\mathbf{C})^{\text {in }}$ that lies over the base point $\boldsymbol{b}=\left\{b_{1}, \ldots, b_{r}\right\} \in \mathcal{U}_{r}$ (from $\S 1.3$ ). We assume here that all $b_{i} \in \mathcal{Q}$. Then $\beta$ fixes all points of $\mathcal{U}^{(r)}$ that lie over $\boldsymbol{b}$, hence $c(\beta)(\boldsymbol{p})=\theta^{-1}\left(\theta\left(\boldsymbol{p}^{\beta^{-1}}\right)\right)^{\beta}=\boldsymbol{p}^{\beta^{-1}}$. The point $\boldsymbol{p}$ corresponds to the $\operatorname{Inn}(G)$-class of some tuple $\left(\sigma_{1}, \ldots, \sigma_{r}\right) \in \mathrm{Ni}(\mathbf{C})$ under the identification from $\S 1.3$. Further, $\boldsymbol{p}^{\beta^{-1}}$ corresponds to the class of a tuple $\left(\rho_{1}, \ldots, \rho_{r}\right)$ with $\rho_{i}$ conjugate $\sigma_{i}^{m}$ for all $i$, where $m$ is given by the condition that $\beta$ acts on the $|G|$-th roots of unity as $\zeta \mapsto \zeta^{m}$ (see $\S 3.2$ below).

Part 1: The case that $\mathbf{C}$ is rationally rigid. This means that the $C_{i}$ are rational conjugacy classes (i.e., $C_{i}^{m}=C_{i}$ for all integers $m$ prime to $\left.|G|\right)$. By the rigidity assumption, it follows that $\left(\sigma_{1}, \ldots, \sigma_{r}\right)$ and $\left(\rho_{1}, \ldots, \rho_{r}\right)$ are conjugate under $\operatorname{Inn}(G)$. That is, $\boldsymbol{p}^{\beta^{-1}}=\boldsymbol{p}$. Then also $c(\beta)(\boldsymbol{p})=\boldsymbol{p}$, hence $c(\beta)$ is the identity (being an automorphism of an unramified cover). Thus $\theta$ is defined over $\mathcal{Q}$. Therefore the covers $\mathcal{H}(\mathbf{C})^{\text {in }} \rightarrow \mathcal{U}_{r}$ and $\mathcal{U}^{(r)} \rightarrow \mathcal{U}_{r}$ are equivalent over $\mathcal{Q}$.

Let $|\chi, h|$ be any point of $\mathcal{H}(\mathbf{C})^{\text {in }}$. It follows from the above and Corollary 1 that the cover $\chi$ together with all its automorphisms can be defined over some field $k$ if and only if the branch points $x_{1}, \ldots, x_{r}$ of $\chi$ are rational over $k$. As usual, then we have a Galois extension of $k(x)$ with group isomorphic to $G$, regular over $k$ and with branch points $x_{1}, \ldots, x_{r}$. This is also the conclusion of Thompson's rigidity criterion [Th].

Part 2: An example with $\mathbf{C}$ rigid, but not rationally rigid. Let $G=\operatorname{PSL}_{2}(p)$ for a prime $p>3$ for which 3 is not a quadratic residue modulo $p$. Let $\mathrm{C}_{1}$ be the conjugacy class of elements of order 3 , and let $\mathrm{C}_{2}$ and $\mathrm{C}_{3}$ be the two conjugacy classes of $G$ of elements of order $p$. Let $r=3$ and $\mathbf{C}=\left(\mathrm{C}_{1}, \mathrm{C}_{2}, \mathrm{C}_{3}\right)$. This triple is rational and rigid [Ma, 1 ; p. 180], but the single classes $C_{2}$ and $C_{3}$ are not rational: An element $\sigma \in G$ of order $p$ is conjugate to $\sigma^{m}$ if and only if $m$ is a quadratic residue $\bmod p$. It follows that $c(\beta)=1$ if and only if $\beta$ acts on the $p$-th roots of unity as $\zeta \rightarrow \zeta^{m}$ with $m$ a quadratic residue $\bmod p$. These $\beta$ form a subgroup $\Gamma$ of $G_{\mathcal{Q}}$ of index 2. $\left(\Gamma=G_{K}\right.$ with $K=\mathcal{Q}\left(\sqrt{(-1)^{(p-1) / 2} p}\right)$. Thus $c$ takes only two values, the non-trivial value being an involution. Since all involutions in $\operatorname{Aut}\left(\mathcal{U}^{(3)} / \mathcal{U}_{3}\right) \cong S_{3}$ are conjugate, it follows that the space $\mathcal{H}(\mathbf{C})^{\text {in }}$ is $\mathcal{Q}$-isomorphic to that $\mathcal{Q}$-form of $\mathcal{U}^{(3)}$ given by the following action of $G_{\mathcal{Q}}$ on the $\overline{\mathcal{Q}}$-points: Each $\beta \in \Gamma$ sends $\left(x_{1}, x_{2}, x_{3}\right)$ to $\left(x_{1}^{\beta}, x_{2}^{\beta}, x_{3}^{\beta}\right)$, and each $\beta \in G_{\mathcal{Q}} \backslash \Gamma$ sends $\left(x_{1}, x_{2}, x_{3}\right)$ to $\left(x_{1}^{\beta}, x_{3}^{\beta}, x_{2}^{\beta}\right)$.

\section{§2.2. Irreducibility of Hurwitz spaces, and full high branching:}

Corollary 1 shows that to go further with the inverse Galois problem we need to find $\mathcal{Q}$-points on $\mathcal{H}^{\text {in }}$. These $\mathcal{Q}$-points lie on absolutely irreducible components of $\mathcal{H}^{\text {in }}$ that are defined over $\mathcal{Q}$. The first step, therefore, is to find such components of $\mathcal{H}^{\text {in }}$. Theorem 1 gives a criterion to decide when a Hurwitz subspace $\mathcal{H}(\mathbf{C})^{\text {in }}$ of $\mathcal{H}^{\text {in }}$ is such a component. To apply this criterion, the following group-theoretic condition is crucial:

(*) The Schur multiplier of $G$ is generated by commutators (cf. $\S 2.4$ ). 
An (unpublished) theorem of Conway and Parker [CP] shows that the Hurwitz group $H_{r}$ acts transitively on $\mathrm{Ni}(\mathbf{C})^{\text {in }}$ if $(*)$ holds and the $r$-tuple $\mathbf{C}$ contains each non-trivial conjugacy class of $G$ a suitably large number of times. For the convenience of the reader, we supply a proof of this in the Appendix, adapted from [CP]. It follows from Theorem 1 that the Hurwitz space $\mathcal{H}=\mathcal{H}(\mathbf{C})^{\text {in }}$ is absolutely irreducible under the above conditions. If, in addition, $\mathbf{C}$ is rational and $\mathrm{Ni}(\mathbf{C})^{\mathrm{in}}$ is non-empty (this holds, for example, if each non-trivial conjugacy class of $G$ occurs the same - suitably large - number of times in $\mathbf{C})$, then the corresponding Hurwitz space is the desired absolutely irreducible $\mathcal{Q}$-component of $\mathcal{H}^{\text {in }}$. Summarizing:

Proposition 1: Assume that $G$ satisfies $(*)$. Then for infinitely many $r$, there exists an $r$-tuple $\mathbf{C}$ of conjugacy classes of $G$ with the following property: The Hurwitz space $\mathcal{H}(\mathbf{C})^{\text {in }}$ is an absolutely irreducible component of $\mathcal{H}_{r}^{\mathrm{in}}(G)$ that is defined over $\mathcal{Q}$. In particular, the latter is true if $\mathbf{C}$ is rational, $\mathrm{Ni}(\mathbf{C})^{\mathrm{in}}$ is non-empty and $\mathbf{C}$ contains each non-trivial conjugacy class of $G$ a suitably large number of times.

In Lemma 2 below we show that each finite group $H$ is the quotient of a finite group $G$ that satisfies $\left(^{*}\right)$ and has trivial center. Thus, in order to demonstrate that $H$ is regular over $\mathcal{Q}$, by Corollary 1 it suffices to show the following: One of the absolutely irreducible Hurwitz spaces $\mathcal{H}$ defined over $\mathcal{Q}$ that are associated to $G$ has a $\mathcal{Q}$ - rational point. At this time we don't know how to get a rational point on $\mathcal{H}$. But, as far as we know, it is even possible that for suitably large $r, \mathcal{H}$ is a unirational variety. In this case, it has a dense set of rational points.

It seems natural to conjecture that if a group $G$ can be realized as $G(L / \mathcal{Q}(x))$ with $L$ regular over $\mathcal{Q}$, then in fact there are such realizations with an arbitrarily large number of branch points (i.e., $\mathcal{H}_{r}^{\text {in }}(G)$ has $\mathcal{Q}$-points for infinitely many $r$ ). Let $\chi: X \rightarrow \mathcal{P}^{1}$ be a cover with $r$ branch points corresponding to the function field extension $L / \mathcal{Q}(x)$, let $h: G(L / \mathcal{Q}(x)) \rightarrow G$ be an isomorphism, and let $\boldsymbol{p}^{\prime} \in \mathcal{H}_{r}^{\text {in }}(G)$ be the point corresponding to the pair $(\chi, h)$. To each branch point of the cover $\chi$ there is associated a conjugacy class of $G$ (via the isomorphism $h$ ), represented by the corresponding branch cycle. The conjugacy classes of $G$ arising that way are exactly those occurring in the $r$-tuple $\mathbf{C}$ with $\boldsymbol{p}^{\prime} \in \mathcal{H}(\mathbf{C})^{\text {in }}$. We say that $G$ is regular over $\mathcal{Q}$ with full high branching if for each integer $t$ the group $G$ can be realized as $G(L / \mathcal{Q}(x)$ ) (with $L$ regular over $\mathcal{Q}$ ) in such a way that each non-trivial conjugacy class of $G$ is associated to at least $t$ branch points.

For an $r$-tuple $\mathbf{C}$ of conjugacy classes of $G$, let $\tau(\mathbf{C}) \geq 0$ be the minimal number of times that any non-trivial conjugacy class of $G$ occurs in $\mathbf{C}$. We can arrange all the rational tuples $\mathbf{C}$ of non-trivial conjugacy classes of $G$ in a doubly indexed collection $\left\{\mathbf{C}_{t i}\right\}_{t \in \mathcal{N}, i \in I_{t}}$ with $\tau\left(\mathbf{C}_{t i}\right)=t$. From Corollary 1 and Proposition 1 we get the following result:

Proposition 2: Suppose $G$ satisfies $\left(^{*}\right)$ and has trivial center. Then for suitably large $t$, the Hurwitz spaces $\mathcal{H}\left(\mathbf{C}_{t i}\right)^{\text {in }}$ are absolutely irreducible varieties defined over $\mathcal{Q}$. And $G$ is regular over $\mathcal{Q}$ with full high branching (as defined above) if and only if for infinitely many values of there exist such Hurwitz spaces $\mathcal{H}\left(\mathbf{C}_{t i}\right)^{\text {in }}$ that have $\mathcal{Q}$-points.

$\S$ 2.3. The application to PAC-fields: A field $P$ is called $\mathrm{P}$ (seudo)A(lgebraically)C(losed) if every absolutely irreducible variety defined over $P$ has a $P$-rational point. Consider a PAC-field $P$ of characteristic 0 . It follows from Corollary 1 and Proposition 1 that every finite group $G$ satisfying $\left(^{*}\right)$ and having trivial center is regular over $P$. But by Lemma 2 below the quotients of these groups $G$ yield all finite groups. Thus we get the following result.

Theorem 2: If $P$ is a $P A C$-field of characteristic 0 , then every finite group is regular over $P$. In particular, if $P$ is also Hilbertian, then every finite group is a Galois group over $P$.

PAC-fields first appeared in $[\mathrm{Ax}]$ and have been studied since then by various authors (cf. [FrJ]). PAC fields have projective absolute Galois group - a result of Ax [FrJ; Theorem 10.17]. Conversely, if $H$ is a projective profinite group, then there exists a PAC field $P$ such that $H$ is the absolute Galois group of $P$-an observation of Lubotzky and van den Dries ([LD], [FrJ; Corollary 20.16]). 
There are many examples of Hilbertian PAC fields inside of $\overline{\mathcal{Q}}$. For example, F. Pop $[\mathrm{P}]$ has recently announced that one obtains a PAC-field by adjoining $\sqrt{-1}$ to the field of all totally real algebraic numbers. This PAC-field is Hilbertian by Weissauer's theorem [Ws] (which says that any proper finite extension of a Galois extension of a Hilbertian field is Hilbertian). Thus Theorem 2 applies to it. Furthermore, there are PAC-fields $P$ with the property that they are Galois over $\mathcal{Q}$, and $G(P / \mathcal{Q}) \cong \prod_{n=2}^{\infty} S_{n}$ [FrJ; p. 224, Theorem 16.46]. Again these are also Hilbertian by Weissauer's theorem.

On the other hand, the abelian closure of any number field has projective absolute Galois group and it is Hilbertian [FrJ; Theorem 15.6]. But Frey noted that such a field isn't PAC ([Fy] or [FrJ; Corollary 10.15]). Shafarevich conjectured that the abelian closure of the rationals has an $\omega$-free absolute Galois group. Our methods yield an analogue of this (to appear in a sequel to the present paper): Every countable Hilbertian PAC-field of characteristic 0 has an $\omega$-free absolute Galois group (cf. the Introduction). Now let $\mathcal{F}_{p}$ denote the finite field with $p$ elements.

Corollary 2: Let $G$ be any finite group. Then $G$ is regular over $\mathcal{F}_{p}$ for all but finitely many primes $p$.

Corollary 2 can be derived from Theorem 2 as follows: Assume the claim is wrong for infinitely many primes $p$. Then there exists a non-principal ultraproduct $P$ of the fields $\mathcal{F}_{p}$ for these primes $p$. The field $P$ is a PAC-field by a result of Ax [FrJ; Cor. 10.6] (this was the original motivation for the introduction of PAC-fields). Also, $\operatorname{char}(P)=0$. Thus $G$ is regular over $P$ by Theorem 2. It is easy to give first order quantified statements equivalent to the statement that $G$ is regular over a given field; it is an elementary statement. Thus it follows that $G$ is regular over some (in fact, infinitely many) of the fields $\mathcal{F}_{p}$ occurring in the above ultraproduct - a contradiction. We omit the details, and rather give the following more direct proof. The underlying idea is the same as for the proof that the above ultraproduct is PAC; it uses the existence of $\mathcal{F}_{p}$-rational points on absolutely irreducible varieties defined over $\mathcal{F}_{p}$, for sufficiently large $p$ (the Lang-Weil observation). First we need a basic lemma:

Lemma 0: Suppose $R$ is an integral domain, and $k$ is its field of fractions. Let $L / k(x)$ be a finite Galois extension, regular over $k$. Then there is some $u \neq 0$ in $R$ with the following property: For any field $k^{\prime}$ such that $R$ admits a homomorphism $\lambda: R \rightarrow k^{\prime}$ with $\lambda(u) \neq 0$, there is a Galois extension $L^{\prime} / k^{\prime}\left(x^{\prime}\right)$ with $x^{\prime}$ transcendental over $k^{\prime}$ and $L^{\prime}$ regular over $k^{\prime}$ and $G\left(L^{\prime} / k^{\prime}\left(x^{\prime}\right)\right) \cong G(L / k(x))$.

Proof: Choose $y_{1} \in L$ with $L=k\left(x, y_{1}\right)$. Then there is a polynomial $f \in R[x, y]$ of degree $n=[L: k(x)]$ in $y$, such that $f\left(x, y_{1}\right)=0$. By multiplying $y_{1}$ by the $y^{n}$-coefficient of $f$ we may assume that $f$ is monic in $y$. Then $f$ is absolutely irreducible (as a polynomial in two variables over $k$ ) since $L$ is regular over $k$.

By the Bertini-Noether theorem (e.g., [FrJ, Prop. 8.8]) there is some $u_{0} \neq 0$ in $R$ with this property: If $\lambda$ is a homomorphism from $R$ to a field $k^{\prime}$ with $\lambda\left(u_{0}\right) \neq 0$, then the image $f^{\prime} \in k^{\prime}[x, y]$ of $f$ under $\lambda$ is again absolutely irreducible. From now on we assume that this condition $\lambda\left(u_{0}\right) \neq 0$ holds.

The map $\lambda$ extends canonically to a map $R[x] \rightarrow k^{\prime}\left[x^{\prime}\right]$, sending $x$ to some element $x^{\prime}$ that is transcendental over $k^{\prime}$. This map can further be extended to a place of $L$ : a homomorphism $\bar{\lambda}$ from some valuation ring $P$ of $L$ (containing $R[x]$ ) into the algebraic closure of $k^{\prime}\left(x^{\prime}\right)$. Since $P$ is integrally closed and $f(x, y)$ is monic in $y$, the elements $y_{1}, \ldots, y_{n} \in L$ with $f\left(x, y_{i}\right)=0$ lie in $P$. Set $L^{\prime}=k^{\prime}\left(x^{\prime}, y_{1}^{\prime}, \ldots, y_{n}^{\prime}\right)$, where $y_{i}^{\prime}=\bar{\lambda}\left(y_{i}\right)$.

Since $f^{\prime}\left(x^{\prime}, y_{i}^{\prime}\right)=0$ and $f^{\prime}$ is absolutely irreducible, the element $y_{i}^{\prime}$ has degree $n$ over $k^{\prime}\left(x^{\prime}\right)$. Hence for any $b \in R\left[x, y_{i}\right]$ we have: $\bar{\lambda}(b)=0$ if and only if $\lambda$ annihilates all coefficients in the unique expression of $b$ as an $R$-linear combination of the $x^{\nu} y_{i}^{\mu}$ with $\nu \geq 0,0 \leq \mu \leq n-1$. We will refer to this fact as the criterion $(\mathrm{C})$.

Since $L$ is the field of fractions of $S_{1} \stackrel{\text { def }}{=} R\left[x, y_{1}\right]$, there is $c \neq 0$ in $S_{1}$ such that $c y_{i} \in S_{1}$ for all $i=1, \ldots, n$. Then $b=c \prod_{i \neq j}\left(c y_{i}-c y_{j}\right)$ is a non-zero element of $S_{1}$, and $b y_{i} \in S_{1}$ for all $i$. By criterion (C), we can multiply $u_{0}$ by certain elements of $R$ to obtain some $u \neq 0$ in $R$ with the following property: If $\lambda(u) \neq 0$ then $\bar{\lambda}(\sigma(b)) \neq 0$ for all $\sigma \in G(L / k(x))$.

We will show that $u$ is as desired. So assume $\lambda(u) \neq 0$. Then $y_{i}^{\prime}=\bar{\lambda}(b)^{-1} \bar{\lambda}\left(b y_{i}\right) \in k^{\prime}\left(x^{\prime}, y_{1}^{\prime}\right)$ for all $i$. Therefore $L^{\prime}=k^{\prime}\left(x^{\prime}, y_{1}^{\prime}, \ldots, y_{n}^{\prime}\right)=k^{\prime}\left(x^{\prime}, y_{1}^{\prime}\right)$. Thus $\left[L^{\prime}: k^{\prime}\left(x^{\prime}\right)\right]=n$, and $L^{\prime}$ is regular over $k^{\prime}$ (since $f^{\prime}$ is absolutely irreducible). Furthermore, since $\bar{\lambda}(b) \neq 0$, the $y_{1}^{\prime}, \ldots, y_{n}^{\prime}$ are pairwise distinct. 
It remains to show that $L^{\prime}$ is Galois over $k^{\prime}\left(x^{\prime}\right)$ with Galois group isomorphic to $G \stackrel{\text { def }}{=} G(L / k(x))$. The $\operatorname{ring} S=R\left[x, y_{1}, \ldots, y_{n}\right]$ is clearly invariant under $G$. We claim that also $S \cap \operatorname{ker}(\bar{\lambda})$ is $G$-invariant: Namely, for each $s \in S$ the element $s_{1}=b^{m} s$ lies in $S_{1}$ for some positive integer $m$. If $\bar{\lambda}(s)=0$ then $\bar{\lambda}\left(s_{1}\right)=0$. By criterion (C) it follows that $\bar{\lambda}\left(\sigma\left(s_{1}\right)\right)=0$ for each $\sigma \in G$. Thus $\bar{\lambda}(\sigma(b))^{m} \bar{\lambda}(\sigma(s))=0$, which implies $\bar{\lambda}(\sigma(s))=0$.

It follows that $G$ acts naturally on $\bar{\lambda}(S)$. This yields a homomorphism $G \rightarrow \operatorname{Aut}\left(L^{\prime} / k^{\prime}\left(x^{\prime}\right)\right)$, which is injective since $G$ acts transitively on $y_{1}^{\prime}, \ldots, y_{n}^{\prime}$ (and $|G|=n$ ). Thus $\left|\operatorname{Aut}\left(L^{\prime} / k^{\prime}\left(x^{\prime}\right)\right)\right| \geq n=\left[L^{\prime}: k^{\prime}\left(x^{\prime}\right)\right]$, which shows that $L^{\prime}$ is Galois over $k^{\prime}\left(x^{\prime}\right)$ with group isomorphic to $G$.

Proof of Corollary 2: By Lemma 2 we may assume that $G$ satisfies $\left(^{*}\right)$ and has trivial center.

Part 1: Here we show that there exists a finite extension $k^{\prime} / \mathcal{Q}(t)$, with $t$ transcendental over $\mathcal{Q}$ and $k^{\prime}$ regular over $\mathcal{Q}$, and a Galois extension $L^{\prime} / k^{\prime}(x)$, regular over $k^{\prime}$ and with Galois group isomorphic to $G$.

By Proposition 1 there exists $r \geq 3$ such that the space $\mathcal{H}_{r}^{\text {in }}(G)$ has an absolutely irreducible component $\mathcal{H}$ defined over $\mathcal{Q}$. Let $\boldsymbol{p}$ be a generic point of $\mathcal{H}$ over $\mathcal{Q}$, and consider $k \stackrel{\text { def }}{=} \mathcal{Q}(\boldsymbol{p})=\mathcal{Q}(\mathcal{H})$. Corollary 1 gives a Galois extension $L / k(x)$, regular over $k$, with Galois group isomorphic to $G$. Let $R \subset k$ be the coordinate ring of an affine open subset $\mathcal{H}_{0}$ of $\mathcal{H}$ defined over $\mathcal{Q}$. Let $u$ be a non-zero element of $R$ with the properties from Lemma 0 , and let $\mathcal{H}_{1}$ be the complement of the vanishing set of $u$ in $\mathcal{H}_{0}$. By [FrJ, Cor. 9.32] there exists an absolutely irreducible curve $C$ on $\mathcal{H}_{1}$ defined over $\mathcal{Q}$. The restriction homomorphism $\lambda: R \rightarrow k^{\prime}$, where $k^{\prime}=\mathcal{Q}(C)$, satisfies $\lambda(u) \neq 0$. Therefore, by Lemma 0 there exists a Galois extension $L^{\prime} / k^{\prime}(x)$ with the desired properties. (Indeed, $k^{\prime}$ is regular over $\mathcal{Q}$ because $C$ is absolutely irreducible).

Part 2: Reduction mod $p$. As in the proof of Lemma 0 we have $k^{\prime}=\mathcal{Q}(t, z)$ with $f(t, z)=0$, where $f \in \mathcal{Z}[T, Z]$ is an absolutely irreducible polynomial, monic and of degree $m=\left[k^{\prime}: \mathcal{Q}(t)\right]$ in $Z$. By BertiniNoether, $f$ remains absolutely irreducible modulo $p$ for all but finitely many primes $p$. Thus it follows from the Lang-Weil estimate for the number of rational points on a curve over a finite field (e.g., [FrJ, Th. 3.14]) that the number of pairs $(a, b) \in\left(\mathcal{F}_{p}\right)^{2}$ with $f(a, b)=0$ goes to infinity as $p \rightarrow \infty$.

Now let $u$ be an element of $R=\mathcal{Z}[t, z] \subset k^{\prime}$ with the properties from Lemma 0 (with respect to the extension $L^{\prime} / k^{\prime}(x)$ constructed in Part 1). We have $u=g(t, z)$ for a unique polynomial $g \in \mathcal{Z}[T, Z]$ of degree $<m$ in $Z$. By Bezout's theorem, the number of common solutions of $f(a, b)=0$ and $g(a, b)=0$ over $\mathcal{F}_{p}$ is bounded independent of $p$ (since $f$ is absolutely irreducible and $g \not \equiv 0(\bmod p)$ for all but finitely many $p)$. Hence for all but finitely many primes $p$ there exists $\left(a_{0}, b_{0}\right) \in\left(\mathcal{F}_{p}\right)^{2}$ with $f\left(a_{0}, b_{0}\right)=0$ and $g\left(a_{0}, b_{0}\right) \neq 0$. Such a point $\left(a_{0}, b_{0}\right)$ yields a homomorphism $\lambda: R \rightarrow \mathcal{F}_{p}$ (sending $t$ to $a_{0}$ and $z$ to $\left.b_{0}\right)$ with $\lambda(u) \neq 0$. Now the claim follows from Lemma $0 . \quad \square$

§2.4. Two group-theoretic Lemmas: The goal of this section is to prove Lemma 2 below: Every finite group is the quotient of a finite group $G$ with trivial center satisfying $\left(^{*}\right)$. These conditions were needed above to guarantee the existence of absolutely irreducible $\mathcal{Q}$-components of the parameter space $\mathcal{H}^{\text {in }}$.

For any finite group $G$ let $G^{\prime}$ denote its commutator subgroup and $Z(G)$ its center. Recall that a representation group $R$ of $G$ is a group of maximal order with the property that $R$ has a subgroup $M \subseteq R^{\prime} \cap Z(R)$ satisfying $R / M \cong G$. Such an $R$ always exists (but it is not necessarily unique). The group $M$ is isomorphic to the Schur multiplier $M(G)=H^{2}\left(G, \mathcal{C}^{*}\right)$ of $G$ [Hu; p. 631]. We say that $M(G)$ is generated by commutators (condition (*)) if $M \cap\left\{g^{-1} h^{-1} g h \mid g, h \in R\right\}$ generates $M$. This is independent of the choice of $R$. Our first lemma shows that representation groups satisfy this condition.

Lemma 1: If $H$ is a finite group and $G$ is a representation group of $H$, then the Schur multiplier $M(G)$ is generated by commutators.

Proof: We have a surjection $G \rightarrow H$ with kernel $M_{H} \cong M(H)$ satisfying

$M_{H} \leq G^{\prime} \cap Z(G)$. Let $R$ be a representation group of $G$. Then we have a surjection $R \rightarrow G$ with kernel $M \cong M(G)$ satisfying $M \leq R^{\prime} \cap Z(R)$. Let $C$ be the subgroup of $M$ generated by commutators (from $R$ ), and consider $\bar{R} \stackrel{\text { def }}{=} R / C$. Then $\bar{M} \stackrel{\text { def }}{=} M / C$ contains no non-trivial commutators (from $\bar{R}$ ), and $\bar{M} \leq \bar{R}^{\prime} \cap Z(\bar{R})$.

The map $R \rightarrow G$ induces a map $\varphi: \bar{R} \rightarrow G$ with kernel $\bar{M}$. From $M_{H} \leq G^{\prime}$ we get $L \stackrel{\text { def }}{=} \varphi^{-1}\left(M_{H}\right) \leq$ $\bar{R}^{\prime} \bar{M}=\bar{R}^{\prime}$. Clearly, $\bar{R} / L \cong H$. Furthermore, $[\bar{R}, L] \subset \bar{M}$. Since $\bar{M}$ contains no non-trivial commutators, $[\bar{R}, L]=1$. Therefore, $\bar{R}$ is a central extension of $H$ with kernel $L \leq \bar{R}^{\prime}$. It follows that $|L| \leq|M(H)|$. That is, $\bar{M}=1$ and $M=C$. This proves the lemma. 
Lemma 2: Every finite group $H$ is the quotient of a finite group $G$ with trivial center such that the Schur multiplier of $G$ is generated by commutators (Condition $\left(^{*}\right)$ ).

Proof: By Lemma 1 we may assume that $H$ already has property $(*)$ (Replacing $H$ by a representation group of $H$ ). Let $m=|H|$, let $S$ be a non-abelian finite simple group with trivial Schur multiplier (e.g., $S=\mathrm{SL}_{2}(8)$, see [Hu, Satz 25.7]) and set $A=S^{m}$, the direct product of $m$ copies of $S$. The (regular) wreath-product $G$ of $S$ and $H$ is defined to be the semi-direct product $G=A \times{ }^{s} H$ where $H$ acts on $A$ by permuting the factors of $A$ sharply transitively (i.e., in its regular permutation representation). Clearly $G$ has trivial center.

Any central extension of $S$ splits because $M(S)=1$. By a simple induction argument, it follows that also every central extension of $A=S^{m}$ splits. This implies that every representation group of $G$ has a normal subgroup isomorphic to $A$ such that the quotient by this subgroup is a representation group of $H$. Therefore, $M(G) \cong M(H)$ is generated by commutators.

\section{PROOF OF THEOREM 1 UNDER A CONTINUITY ASSUMPTION}

Assume the hypotheses of Theorem 1 . The unramified coverings $\Psi: \mathcal{H}^{\text {ab }} \rightarrow \mathcal{U}_{r}$ and $\Psi^{\prime}: \mathcal{H}^{\text {in }} \rightarrow \mathcal{U}_{r}$ equip the spaces $\mathcal{H}^{\text {ab }}$ and $\mathcal{H}^{\text {in }}$ with a unique structure as (non-singular, usually reducible) algebraic varieties defined over $\mathcal{C}$ (compatible with their analytic structure) such that the maps $\Psi$ and $\Psi^{\prime}$ are algebraic morphisms defined over $\mathcal{C}$. This follows from the generalized Riemann existence theorem (see [SGA1, exp. XII, Th. 5.1]). By [Se, Thm. 6.7], the field $\mathcal{C}$ can be replaced by $\overline{\mathcal{Q}}$ in the above statement.

From now on we view the spaces $\mathcal{H}^{\text {ab }}$ and $\mathcal{H}^{\text {in }}$ as equipped with this natural structure of a variety defined over $\overline{\mathcal{Q}}$. The resulting notion of $\overline{\mathcal{Q}}$-points of $\mathcal{H}^{\text {ab }}$ and $\mathcal{H}^{\text {in }}$ is clearly compatible with the definition in $\S 2.1$. Furthermore, $\Lambda: \mathcal{H}^{\text {in }} \rightarrow \mathcal{H}^{\text {ab }}$ becomes a morphism defined over $\overline{\mathcal{Q}}$. For each automorphism $\beta$ of $\mathcal{C}$ let $\epsilon_{\beta}:\left(\mathcal{H}^{\mathrm{ab}}\right)^{\beta} \rightarrow \mathcal{H}^{\mathrm{ab}}$ be the map sending $|\varphi|^{\beta}$ to $\left|\varphi^{\beta}\right|$ (where $|\varphi| \in \mathcal{H}^{\mathrm{ab}}$ ). Then $\epsilon_{\beta}$ is well-defined (cf. $\S 2.1$ ) and bijective, and we have $\Psi \circ \epsilon_{\beta}=\Psi^{\beta}$.

Similarly, let $\epsilon_{\beta}^{\prime}:\left(\mathcal{H}^{\text {in }}\right)^{\beta} \rightarrow \mathcal{H}^{\text {in }}$ be the map sending $|\chi, h|^{\beta}$ to $\left|\chi^{\beta}, h \circ \beta^{-1}\right|$ (where $|\chi, h| \in \mathcal{H}^{\text {in }}$ ). Also $\epsilon_{\beta}^{\prime}$ is bijective, and $\Psi^{\prime} \circ \epsilon_{\beta}^{\prime}=\left(\Psi^{\prime}\right)^{\beta}$. In the remainder of $\S 3$ we show that Theorem 1 holds under the assumption that the maps $\epsilon_{\beta}$ and $\epsilon_{\beta}^{\prime}$ are continuous (in the complex topology). In $\S 4$ this assumption is verified in the special case that $U$ is self-normalizing in $G$. In $\S 5$ we reduce the problem to this special case.

§3.1. The $\mathcal{Q}$-structure on $\mathcal{H}^{\mathrm{ab}}$ and $\mathcal{H}^{\mathrm{in}}$ : Assume that the map $\epsilon_{\beta}:\left(\mathcal{H}^{\mathrm{ab}}\right)^{\beta} \rightarrow \mathcal{H}^{\mathrm{ab}}$ is continuous (in the complex topology) for each $\beta \in \operatorname{Aut}(\mathcal{C})$. Since $\epsilon_{\beta}$ is bijective and $\Psi \circ \epsilon_{\beta}=\Psi^{\beta}$, it follows that $\epsilon_{\beta}$ is a complex analytic isomorphism, inducing an equivalence between the coverings $\Psi$ and $\Psi^{\beta}$. By the uniqueness of the algebraic structure of $\mathcal{H}^{\text {ab }}$, the map $\epsilon_{\beta}$ is even an algebraic isomorphism defined over $\overline{\mathcal{Q}}$. Further, $\epsilon_{\beta}$ depends only on the restriction of $\beta$ to $\overline{\mathcal{Q}}$ (since the $\overline{\mathcal{Q}}$-points are dense on $\mathcal{H}^{\text {ab }}$, and the corresponding covers $\varphi$ can be defined over $\overline{\mathcal{Q}}$, see $\S 2.1$ ). Thus we use the notation $\epsilon_{\beta}$ also for $\beta \in G_{\mathcal{Q}}$. (Because our maps act from the left, we use the convention $\varphi^{\alpha \beta}=\left(\varphi^{\beta}\right)^{\alpha}$.) It is straightforward to check that the $\epsilon_{\beta}\left(\beta \in G_{\mathcal{Q}}\right)$ satisfy Weil's cocycle condition:

$$
\epsilon_{\alpha} \circ \epsilon_{\beta}^{\alpha}\left(|\varphi|^{\alpha \beta}\right)=\epsilon_{\alpha}\left(\left(\epsilon_{\beta}\left(|\varphi|^{\beta}\right)\right)^{\alpha}\right)=\epsilon_{\alpha}\left(\left|\varphi^{\beta}\right|^{\alpha}\right)=\left|\varphi^{\alpha \beta}\right|=\epsilon_{\alpha \beta}\left(|\varphi|^{\alpha \beta}\right) \quad\left(\alpha, \beta \in G_{\mathcal{Q}}\right)
$$

Hence $\mathcal{H}^{\text {ab }}$ can be defined over $\mathcal{Q}$ such that $\epsilon_{\beta}: \mathcal{H}^{\text {ab }}=\left(\mathcal{H}^{\text {ab }}\right)^{\beta} \rightarrow \mathcal{H}^{\text {ab }}$ is the identity. Since $\Psi \circ \epsilon_{\beta}=\Psi^{\beta}$ it follows that also $\Psi: \mathcal{H}^{\text {ab }} \rightarrow \mathcal{U}_{r}$ is defined over $\mathcal{Q}$. Further it is clear that condition (a) of Theorem 1 holds.

Assuming that $\epsilon_{\beta}^{\prime}:\left(\mathcal{H}^{\text {in }}\right)^{\beta} \rightarrow \mathcal{H}^{\text {in }}$ is continuous (in the complex topology) for each $\beta \in \operatorname{Aut}(\mathcal{C})$, we conclude similarly that $\Psi^{\prime}: \mathcal{H}^{\text {in }} \rightarrow \mathcal{U}_{r}$ can be defined over $\mathcal{Q}$ such that condition (b) of Theorem 1 holds.

§3.2. The remaining part of Theorem 1: We have shown that the spaces $\mathcal{H}^{\text {ab }}$ and $\mathcal{H}^{\text {in }}$ are equipped with a $\mathcal{Q}$-variety structure such that (a) and (b) of Theorem 1 hold. Then also $\Lambda: \mathcal{H}^{\text {in }} \rightarrow \mathcal{H}^{\text {ab }}$ is defined over $\mathcal{Q}$ (c.f. $§ 5.1)$.

Further, the connected components (in the complex topology) of $\mathcal{H}^{\text {ab }}$ and $\mathcal{H}^{\text {in }}$ are irreducible, since they are complex (nonsingular) manifolds. In $\S 1.3$ we have set up a 1-1 correspondence between the components of $\mathcal{H}^{\text {in }}=\mathcal{H}_{r}^{\text {in }}(G)$ and the orbits of the Hurwitz monodromy group $H_{r}$ on the set $\mathcal{E}_{r}^{\text {in }}=\mathcal{E}_{r}^{\text {in }}(G)$. The last assertion in Theorem 1 is a special case of this. 
It remains to show that the Hurwitz space $\mathcal{H}(\mathbf{C})^{\text {in }}$ is defined over $\mathcal{Q}$ if and only if the $r$-tuple $\mathbf{C}=$ $\left(\mathrm{C}_{1}, \ldots, \mathrm{C}_{r}\right)$ of conjugacy classes of $G$ is rational. Consider the group $\Gamma_{0}=\pi_{1}\left(\mathcal{P}^{1} \backslash \boldsymbol{b}, b_{0}\right)=\left\langle\gamma_{1}, \ldots, \gamma_{r}\right\rangle$ as in $\S 1.3$, and let $\boldsymbol{p}=|\chi, h|$ be a point in $\left(\Psi^{\prime}\right)^{-1}(\boldsymbol{b})$. As usual, we write $\chi: \hat{X} \rightarrow \mathcal{P}^{1}$. Let $\iota: \Gamma_{0} \rightarrow$ $\operatorname{Aut}\left(\hat{X} / \mathcal{P}^{1}\right)$ be the surjection from $\S 1.2$ (canonical up to inner automorphisms), and set $f=h \circ \iota, \tau_{i}=\iota\left(\gamma_{i}\right)$ for $i=1, \ldots, r$. Then under the bijection from $\S 1.3$, the point $\boldsymbol{p}$ corresponds to the class of $\left(\sigma_{1}, \ldots, \sigma_{r}\right)$ in $\mathcal{E}_{r}^{\text {in }}$, where $\sigma_{i}=f\left(\gamma_{i}\right)=h\left(\tau_{i}\right)$. By definition, $\boldsymbol{p} \in H(\mathbf{C})^{\text {in }}$ if and only if $\left(\sigma_{1}, \ldots, \sigma_{r}\right) \in \mathrm{Ni}(\mathbf{C})$; the latter means that there exists $\pi \in S_{r}$ such that $\sigma_{i} \in \mathrm{C}_{\pi(i)}$ for $i=1, \ldots, r$.

The branch cycle argument from [Fr,1; p. 63] (see also [Ma,1; p. 47]) yields the following: For a fixed $\beta \in \operatorname{Aut}(\mathcal{C})$, let $\iota^{\prime}: \Gamma_{0} \rightarrow \operatorname{Aut}\left(\hat{X}^{\beta} / \mathcal{P}^{1}\right)$ be defined analogously as $\iota$, and set $\tau_{j}^{\prime}=\iota^{\prime}\left(\gamma_{j}\right)$ for $j=1, \ldots, r$. Then the element $\tau_{i}^{\beta} \in \operatorname{Aut}\left(\hat{X}^{\beta} / \mathcal{P}^{1}\right)$ is conjugate to $\left(\tau_{j}^{\prime}\right)^{m}$, where the integer $m$ is given by the condition that $\beta$ acts on the $|G|$-th roots of unity as $\zeta \mapsto \zeta^{m}$, and the index $j$ is given by $\beta\left(b_{i}\right)=b_{j}$. (Recall that by our choice of $\boldsymbol{b}=\left\{b_{1}, \ldots, b_{r}\right\}$ from $\S 1.1, \beta$ permutes $b_{1}, \ldots, b_{r}$.) Choose an integer $n$ with $m n \equiv 1(\bmod |G|)$. Since $\boldsymbol{p}^{\beta}=\left|\chi^{\beta}, h \circ \beta^{-1}\right|$ (assertion (b)), it follows that the element of $\mathcal{E}_{r}^{\text {in }}$ corresponding to $\boldsymbol{p}^{\beta}$ is the class of $\left(h \circ \beta^{-1}\left(\tau_{1}^{\prime}\right), \ldots, h \circ \beta^{-1}\left(\tau_{r}^{\prime}\right)\right)$; this $r$-tuple has the property that its $j$-th entry is conjugate to $\sigma_{i}^{n}$, where $\beta\left(b_{i}\right)=b_{j}$. It follows that $\boldsymbol{p} \in \mathcal{H}(\mathbf{C})^{\text {in }}$ if and only if $\boldsymbol{p}^{\beta} \in \mathcal{H}\left(\mathbf{C}^{n}\right)^{\text {in }}$, where $\mathbf{C}^{n}=\left(C_{1}^{n}, \ldots, C_{r}^{n}\right)$ (as above).

We have proved that $\beta$ maps $\mathcal{H}(\mathbf{C})^{\text {in }}$ to $\mathcal{H}\left(\mathbf{C}^{n}\right)^{\text {in }}$. Thus $\mathcal{H}(\mathbf{C})^{\text {in }}$ is defined over $\mathcal{Q}$ if and only if $\mathcal{H}\left(\mathbf{C}^{n}\right)^{\text {in }}=\mathcal{H}(\mathbf{C})^{\text {in }}$ for all integers $n$ that are prime to $|G|$. This is true if and only if $\operatorname{Ni}\left(\mathbf{C}^{n}\right)=\operatorname{Ni}(\mathbf{C})$ for all $n$ prime to $|G|$. That is, if and only if $\mathbf{C}$ is rational (as defined in $\S 1.1$ ). This concludes the proof of Theorem 1 under the assumption that the maps $\epsilon_{\beta}$ and $\epsilon_{\beta}^{\prime}$ are continuous.

\section{FAMILIES OF COVERS}

In this section we assume that the hypotheses of Theorem 1 hold, and additionally, that $U$ is self-normalizing in $G$. Then, from (1) of $\S 1.2$, each cover $\varphi: X \rightarrow \mathcal{P}^{1}$ representing a point of $\mathcal{H}^{\text {ab }}=\mathcal{H}_{r}^{\text {ab }}(G, U)$ satisfies $\operatorname{Aut}\left(X / \mathcal{P}^{1}\right)=\{1\}$

We construct a family of affine covers that represent the points of $\mathcal{H}_{r}^{\mathrm{ab}}(G, U)$. It is clear how to construct the family locally, and then the condition $\operatorname{Aut}\left(X / \mathcal{P}^{1}\right)=\{1\}$ allows one to glue the local families uniquely. The family of corresponding compact covers was constructed in [Fr,1]. The construction there also applies to inspect the obstruction for the existence of a total representing family for $\mathcal{H}_{r}^{\text {ab }}(G, U)$ even when $\operatorname{Aut}\left(X / \mathcal{P}^{1}\right) \neq\{1\}$ (the obstruction is in the second étale cohomology group of $\mathcal{H}_{r}^{\text {ab }}(G, U)$ with coefficients in the locally constant "center sheaf"), but we don't need this here.

Let $\mathcal{U}$ be the set of all pairs $(\boldsymbol{a}, z) \in \mathcal{U}_{r} \times \mathcal{P}^{1}$ with $z \notin \boldsymbol{a}$. Then $\mathcal{U}$ is a Zariski-open subset of $\mathcal{U}_{r} \times \mathcal{P}^{1}$ defined over $\mathcal{Q}$. For the moment, we view $\mathcal{U}$ only as an open submanifold of $\mathcal{U}_{r} \times \mathcal{P}^{1}$.

$\S 4.1$. The topological construction of the family: Here the term 'covering' denotes an unramified topological covering map (of not necessarily connected spaces). We start with the covering $\Psi: \mathcal{H}^{\text {ab }}=$ $\mathcal{H}_{r}^{\text {ab }}(G, U) \rightarrow \mathcal{U}_{r}$ of $\S 1.2$. Set

$$
\mathcal{M}=\left\{(\boldsymbol{p}, z) \in \mathcal{H}^{\mathrm{ab}} \times \mathcal{P}^{1}: z \notin \Psi(\boldsymbol{p})\right\} .
$$

Then $\mathcal{M}$ is an open subspace of $\mathcal{H}^{\text {ab }} \times \mathcal{P}^{1}$, and the map $(\boldsymbol{p}, z) \mapsto(\Psi(\boldsymbol{p}), z)$ yields a covering $F: \mathcal{M} \rightarrow \mathcal{U}$. Let $P: \mathcal{M} \rightarrow \mathcal{H}^{\text {ab }}$ be the projection on the first coordinate.

Consider a point $\boldsymbol{p} \in \mathcal{H}^{\mathrm{ab}}$ and a neighborhood $\mathcal{N}^{(\boldsymbol{p})}=\mathcal{N}\left(\boldsymbol{p} ; D_{1}, \ldots, D_{r}\right)$ of $\boldsymbol{p}$ as in $\S 1.2$. Then $\Psi$ maps $\mathcal{N}^{(\boldsymbol{p})}$ homeomorphically onto the set $\mathcal{V}\left(D_{1}, \ldots, D_{r}\right) \cong D_{1} \times \cdots \times D_{r}$ of all $\boldsymbol{a} \in \mathcal{U}_{r}$ with $\left|\boldsymbol{a} \cap D_{i}\right|=1$ for $i=1, \ldots, r$. In particular, $\mathcal{N}^{(\boldsymbol{p})}$ is contractible. Furthermore, $F$ maps the $\operatorname{set} \mathcal{M}\left(\boldsymbol{p} ; D_{1}, \ldots, D_{r}\right)=$ $\mathcal{M}^{(\boldsymbol{p})} \stackrel{\text { def }}{=} P^{-1}\left(\mathcal{N}^{(\boldsymbol{p})}\right)$ homeomorphically onto the set of all $(\boldsymbol{a}, z) \in \mathcal{U}$ with $\boldsymbol{a} \in \mathcal{V}\left(D_{1}, \ldots, D_{r}\right)$. Thus $\mathcal{M}^{(\boldsymbol{p})}$ contains the set $\mathcal{N}^{(\boldsymbol{p})} \times \mathcal{P}^{1} \backslash\left(D_{1} \cup \cdots \cup D_{r}\right)$ as a deformation retract. Choose a base point $a_{0} \in \mathcal{P}^{1} \backslash\left(D_{1} \cup\right.$ $\left.\cdots \cup D_{r}\right)$. We have canonical isomorphisms

$$
\begin{aligned}
\pi_{1}\left(\mathcal{P}^{1} \backslash \Psi(\boldsymbol{p}), a_{0}\right) & \cong \pi_{1}\left(\mathcal{P}^{1} \backslash\left(D_{1} \cup \cdots \cup D_{r}\right), a_{0}\right) \\
\pi_{1}\left(\mathcal{N}^{(\boldsymbol{p})} \times \mathcal{P}^{1} \backslash\left(D_{1} \cup \cdots \cup D_{r}\right),\left(\boldsymbol{p}, a_{0}\right)\right) & \cong \pi_{1}\left(\mathcal{M}^{(\boldsymbol{p})},\left(\boldsymbol{p}, a_{0}\right)\right) .
\end{aligned}
$$


Now let $\varphi: X \rightarrow \mathcal{P}^{1}$ be a cover representing the point $\boldsymbol{p} \in \mathcal{H}^{\mathrm{ab}}$, and let $\left[U_{\varphi}\right]$ be the associated class of subgroups of $\pi_{1}\left(\mathcal{P}^{1} \backslash \Psi(\boldsymbol{p})\right.$ ) (see $\S 1.2$ ). Under the above isomorphisms, [U $U_{\varphi}$ ] corresponds to a class of subgroups of $\pi_{1}\left(\mathcal{M}^{(\boldsymbol{p})},\left(\boldsymbol{p}, a_{0}\right)\right)$, and this class of subgroups gives rise to a covering $\mathcal{T}\left(\boldsymbol{p} ; D_{1}, \ldots, D_{r}\right) \rightarrow$ $\mathcal{M}^{(\boldsymbol{p})}=\mathcal{M}\left(\boldsymbol{p} ; D_{1}, \ldots, D_{r}\right)$. This covering has no non-trivial automorphisms, since $U$ is self-normalizing in $G$ (c.f. (1) in $\$ 1.2$ ).

From the lack of non-trivial automorphisms of the above covering it follows that for each inclusion $\mathcal{M}\left(\boldsymbol{p} ; D_{1}, \ldots, D_{r}\right) \rightarrow \mathcal{M}\left(\boldsymbol{p}^{\prime} ; D_{1}^{\prime}, \ldots, D_{r}^{\prime}\right)$ of two of the above neighborhoods, there is a unique embedding $\mathcal{T}\left(\boldsymbol{p} ; D_{1}, \ldots, D_{r}\right) \rightarrow \mathcal{T}\left(\boldsymbol{p}^{\prime} ; D_{1}^{\prime}, \ldots, D_{r}^{\prime}\right)$ making the following diagram commute (where the vertical arrows are the coverings constructed in the last paragraph):

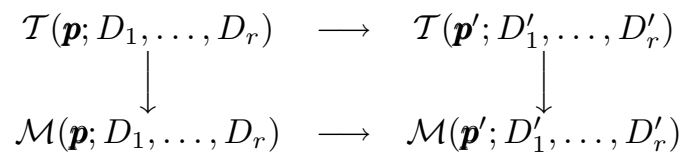

From this uniqueness and the fact that the $\mathcal{N}^{(\boldsymbol{p})}$ form a basis for the topology of $\mathcal{H}^{\text {ab }}$, it follows that the coverings $\mathcal{T}\left(\boldsymbol{p} ; D_{1}, \ldots, D_{r}\right) \rightarrow \mathcal{M}\left(\boldsymbol{p} ; D_{1}, \ldots, D_{r}\right)$ glue together to yield a global covering $\Phi: \mathcal{T} \rightarrow \mathcal{M}$. For each $\boldsymbol{p} \in \mathcal{H}^{\text {ab }}$ denote $(P \circ \Phi)^{-1}(\boldsymbol{p})$ by $\mathcal{T}_{\boldsymbol{p}}$, and let $\Phi_{\boldsymbol{p}}: \mathcal{T}_{\boldsymbol{p}} \rightarrow \mathcal{P}^{1} \backslash \Psi(\boldsymbol{p})$ be the composition of $\Phi$ with projection to $\mathcal{P}^{1}$. By construction it is clear that the covering $\Phi_{\boldsymbol{p}}$ represents the point $\boldsymbol{p}$ of $\mathcal{H}^{\text {ab }}$. Thus we call $\Phi: \mathcal{T} \rightarrow \mathcal{M}$ the (partial) family of all covers parametrized by $\mathcal{H}^{\text {ab }}$. We apply the attribute 'partial' because the fibers $\mathcal{T}_{\boldsymbol{p}}$ do not yield the full compact covers of $\mathcal{P}^{1}$ as discussed at the beginning of $\S 4$.

§4.2. Uniqueness of the family: Assume we have coverings $\Psi^{\prime}: \mathcal{H}^{\prime} \rightarrow \mathcal{U}_{r}$ and $\Phi^{\prime}: \mathcal{T}^{\prime} \rightarrow \mathcal{M}^{\prime}$, where $\mathcal{M}^{\prime}=\left\{(\boldsymbol{q}, z) \in \mathcal{H}^{\prime} \times \mathcal{P}^{1}: z \notin \Psi^{\prime}(\boldsymbol{q})\right\}$. Define the maps $F^{\prime}: \mathcal{M}^{\prime} \rightarrow \mathcal{U}$ and $P^{\prime}: \mathcal{M}^{\prime} \rightarrow \mathcal{H}^{\prime}$ analogously as $F$ and $P$, respectively. Assume further that for each $\boldsymbol{q} \in \mathcal{H}^{\prime}$ the fiber $\mathcal{T}_{\boldsymbol{q}}^{\prime}=\left(P^{\prime} \circ \Phi^{\prime}\right)^{-1}(\boldsymbol{q})$ has the following property: The natural map $\mathcal{T}_{\boldsymbol{q}}^{\prime} \rightarrow \mathcal{P}^{1} \backslash \Psi^{\prime}(\boldsymbol{q})$ is a cover that represents a point $\epsilon(\boldsymbol{q})$ of $\mathcal{H}^{\text {ab }}$. Then $\epsilon: \mathcal{H}^{\prime} \rightarrow \mathcal{H}^{\text {ab }}$ is continuous.

To prove the above, note that $\Psi \circ \epsilon=\Psi^{\prime}$ from the definitions. As $\Psi^{\prime}$ is a covering, any $\boldsymbol{q} \in \mathcal{H}^{\prime}$ has a neighborhood $\mathcal{N}^{\prime}$ such that $\Psi^{\prime}$ maps $\mathcal{N}^{\prime}$ homeomorphically onto some $\mathcal{V}\left(D_{1}, \ldots, D_{r}\right)$ (where $D_{1}, \ldots, D_{r}$ are pairwise disjoint discs on $\mathcal{P}^{1}$ around the elements of $\left.\Psi^{\prime}(\boldsymbol{q})\right)$. Then $F^{\prime}$ maps $\mathcal{M}^{\prime}\left(\boldsymbol{q} ; D_{1}, \ldots, D_{r}\right) \stackrel{\text { def }}{=}\left(P^{\prime}\right)^{-1}\left(\mathcal{N}^{\prime}\right)$ homeomorphically onto $\left\{(\boldsymbol{a}, z) \in \mathcal{U}: \boldsymbol{a} \in \mathcal{V}\left(D_{1}, \ldots, D_{r}\right)\right\}$. Thus $\mathcal{M}^{\prime}\left(\boldsymbol{q} ; D_{1}, \ldots, D_{r}\right)$ contains $\mathcal{N}^{\prime} \times \mathcal{P}^{1} \backslash\left(D_{1} \cup\right.$ $\left.\cdots \cup D_{r}\right)$ as a deformation retract. Since $\mathcal{N}^{\prime}$ is contractible, all covers $\mathcal{T}_{\boldsymbol{q}^{\prime}}^{\prime} \rightarrow \mathcal{P}^{1} \backslash \Psi^{\prime}\left(\boldsymbol{q}^{\prime}\right)$ for $\boldsymbol{q}^{\prime} \in \mathcal{N}^{\prime}$ restrict, up to equivalence, to the same covering of $\mathcal{P}^{1} \backslash\left(D_{1} \cup \cdots \cup D_{r}\right)$. Thus $\epsilon\left(\mathcal{N}^{\prime}\right)=\mathcal{N}\left(\boldsymbol{p} ; D_{1}, \ldots, D_{r}\right)$ for $\boldsymbol{p}=\epsilon(\boldsymbol{q})$. This shows that $\epsilon$ is continuous, even a local homeomorphism.

$\S$ 4.3. The $\epsilon_{\beta}$ are continuous: As in $\S 3$ view $\Psi: \mathcal{H}^{\text {ab }} \rightarrow \mathcal{U}_{r}$ as a morphism of algebraic varieties (defined over $\mathcal{C}$ is enough here). Then also $F: \mathcal{M} \rightarrow \mathcal{U}$ is such a morphism. Similarly, the unramified covering $\Phi: \mathcal{T} \rightarrow \mathcal{M}$ equips $\mathcal{T}$ with a unique variety structure defined over $\mathcal{C}$ such that $\Phi$ becomes an algebraic morphism.

Now consider some $\beta \in \operatorname{Aut}(\mathcal{C})$. It is clear that the maps $\Psi^{\beta}:\left(\mathcal{H}^{\mathrm{ab}}\right)^{\beta} \rightarrow \mathcal{U}_{r}$ and $\Phi^{\beta}: \mathcal{T}^{\beta} \rightarrow \mathcal{M}^{\beta}$ are again coverings. They satisfy the conditions of $\S 4.2$ with $\epsilon=\epsilon_{\beta}$, and so $\epsilon_{\beta}$ is continuous. Thus we have verified the continuity assumption on the $\epsilon_{\beta}$ in the case that $U$ is self-normalizing in $G$.

Remark: With some additional work it is possible to give a direct construction of the variety structure on $\mathcal{H}^{\text {ab }}$, without appealing to the generalized Riemann existence theorem. Consider a point $|\varphi| \in \mathcal{H}^{\text {ab }}$ such that the set $\boldsymbol{a}$ of branch points of $\varphi$ is a generic point of $\mathcal{U}_{r}$ (over $\mathcal{Q}$ ). Define $K$ as the minimal field of definition of $\varphi$. Then the $\mathcal{Q}$-irreducible component of $\mathcal{H}^{\text {ab }}$ containing $|\varphi|$ can be identified with the normalization of the variety $\mathcal{U}_{r}$ in the extension field $K$ of $\mathcal{Q}(\boldsymbol{a})=\mathcal{Q}\left(\mathcal{U}_{r}\right)$. To set up this identification, one again needs the above family $\mathcal{T} \rightarrow \mathcal{M}$, whose respective $\mathcal{Q}$-component can be constructed in that approach as the normalization of the respective component of $\mathcal{M}$ in the field $K(X)$, where $\varphi: X \rightarrow \mathcal{P}^{1}$. This yields the desired variety structure defined over $\mathcal{Q}$ on the spaces $\mathcal{H}_{r}^{\text {ab }}(G, U)$, where $U$ is self-normalizing in $G$. Proceed as in $\S 5$ to transfer this variety structure to the spaces $\mathcal{H}_{r}^{\text {in }}(G)$, and $\mathcal{H}_{r}^{\text {ab }}(G, U)$ for $U$ not self-normalizing. 


\section{CONCLUSION OF THE PROOF OF THEOREM 1}

Assume the hypotheses of Theorem 1 hold. From $\S 3$ it only remains to show that for each automorphism $\beta$ of $\mathcal{C}$ the maps $\epsilon_{\beta}:\left(\mathcal{H}^{\mathrm{ab}}\right)^{\beta} \rightarrow \mathcal{H}^{\text {ab }}$ and $\epsilon_{\beta}^{\prime}:\left(\mathcal{H}^{\text {in }}\right)^{\beta} \rightarrow \mathcal{H}^{\text {in }}$ are continuous.

$\S 5.1$. Some reductions: Here we make use of the covering $\Lambda: \mathcal{H}^{\text {in }} \rightarrow \mathcal{H}^{\text {ab }}$ with $\Psi^{\prime}=\Psi \circ \Lambda$. As noted in $\S 3$, we may view $\Lambda$ as an algebraic morphism (defined over $\mathcal{C}$ is enough here). Let $\beta \in \operatorname{Aut}(\mathcal{C})$. It is immediate from the definition of $\Lambda$ in $\S 1.2$ that

$$
\epsilon_{\beta} \circ \Lambda^{\beta}=\Lambda \circ \epsilon_{\beta}^{\prime} .
$$

This implies:

(5.1) If $\epsilon_{\beta}^{\prime}$ is continuous, then also $\epsilon_{\beta}$ is continuous.

(5.2) Under the hypothesis $\operatorname{Aut}(G, U)=\operatorname{Inn}(G)$, the map $\epsilon_{\beta}$ is continuous if and only if $\epsilon_{\beta}^{\prime}$ is continuous.

Note that if $\operatorname{Aut}(G, U)=\operatorname{Inn}(G)$ then $\mathcal{E}_{r}^{\mathrm{ab}}(G, U)=\mathcal{E}_{r}^{\mathrm{in}}(G)(\S 1.1)$; hence the coverings $\Psi: \mathcal{H}_{r}^{\mathrm{ab}}(G, U) \rightarrow \mathcal{U}_{r}$ and $\Psi^{\prime}: \mathcal{H}_{r}^{\text {in }}(G) \rightarrow \mathcal{U}_{r}$ are equivalent $(\S 1.3)$, and therefore $\Lambda$ is an isomorphism. This proves (5.2).

Combining (5.2) and $\S 4.3$ we get:

(5.3) If $U$ is self-normalizing in $G$ and $\operatorname{Aut}(G, U)=\operatorname{Inn}(G)$, then $\epsilon_{\beta}^{\prime}$ is continuous.

$\S 5.2$. The $\epsilon_{\beta}^{\prime}$ are continuous: By (5.1) it only remains to show that the $\epsilon_{\beta}^{\prime}$ are continuous. We will reduce this to the special case of (5.3), by constructing a finite group $\tilde{G}$ that satisfies the hypothesis of (5.3) and maps surjectively to $G$. First we need to study how the spaces $\mathcal{H}^{\text {in }}$ behave under surjections $\lambda: \widetilde{G} \rightarrow G$ of finite groups.

Let $N$ be the kernel of $\lambda$. Define a map $\Omega$ from $\mathcal{H}_{r}^{\text {in }}(\tilde{G})$ to the disjoint union $\bigcup_{s=2}^{r} \mathcal{H}_{s}^{\text {in }}(G)$ as follows: Suppose that we are given $|\tilde{\chi}, \tilde{h}|$ in $\mathcal{H}_{r}^{\text {in }}(\tilde{G})$, where $\tilde{\chi}: \tilde{X} \rightarrow \mathcal{P}^{1}$ and $\tilde{h}: \operatorname{Aut}\left(\tilde{X} / \mathcal{P}^{1}\right) \rightarrow \tilde{G}$ is an isomorphism. Let $\chi: \hat{X} \stackrel{\text { def }}{=} \tilde{X} / \tilde{h}^{-1}(N) \rightarrow \mathcal{P}^{1}$ be the induced cover and let $h: \operatorname{Aut}\left(\hat{X} / \mathcal{P}^{1}\right) \rightarrow G$ be the isomorphism making the following diagram commute (the left vertical arrow is the canonical map):

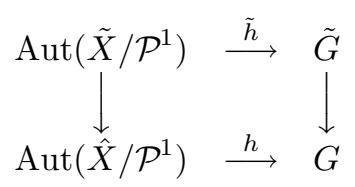

Then $\Omega$ maps $|\tilde{\chi}, \tilde{h}|$ to $|\chi, h|$.

Equip $\bigcup_{s=2}^{r} \mathcal{H}_{s}^{\text {in }}(G)$ with the topology of a disjoint union of open (and closed) spaces. Then $\Omega$ is continuous (easy to check from the set-up of $\S 1.2$ ). Further, the number of branch points of $\Omega(\boldsymbol{p})$ is locally constant for $\boldsymbol{p} \in \mathcal{H}_{r}^{\text {in }}(\tilde{G})$. Thus $\tilde{\mathcal{H}}=\Omega^{-1}\left(\mathcal{H}_{r}^{\text {in }}(G)\right)$ is a union of connected components of $\mathcal{H}_{r}^{\text {in }}(\tilde{G})$. (It could be empty.) Now assume $\tilde{G}$ can be generated by $r-1$ elements. Then a theorem of Gaschütz implies that for each $\left(\sigma_{1}, \ldots, \sigma_{r}\right) \in \mathcal{E}_{r}(G)$ there exists $\left(\tau_{1}, \ldots, \tau_{r}\right) \in \mathcal{E}_{r}(\tilde{G})$ with $\lambda\left(\tau_{i}\right)=\sigma_{i}$ for all $i$ (e.g., [FrJ, Lemma 15.30]). Since $\pi_{1}\left(\mathcal{P}^{1} \backslash \boldsymbol{a}, a_{0}\right)$ is free of rank $r-1$ (where $\boldsymbol{a}=\left\{a_{1}, \ldots, a_{r}\right\}$ as in $\S 1.2$ ), $\Omega$ maps $\mathcal{H}$ surjectively to $\mathcal{H}_{r}^{\text {in }}(G)$. From now on $\Omega: \tilde{\mathcal{H}} \rightarrow \mathcal{H}_{r}^{\text {in }}(G)$ denotes the restriction of the above map to $\tilde{\mathcal{H}}$.

We have $\Psi^{\prime} \circ \Omega=\tilde{\Psi}$, where $\tilde{\Psi}: \tilde{\mathcal{H}} \rightarrow \mathcal{U}_{r}$ is the natural covering that sends $|\tilde{\chi}, \tilde{h}|$ to the set of branch points of $\tilde{\chi}$. Therefore, $\Omega$ is a covering since it is continuous. Again it follows that $\Omega$ is an algebraic morphism (defined over $\mathcal{C}$ ).

Now consider $\beta \in \operatorname{Aut}(\mathcal{C})$. With $\tilde{\epsilon}_{\beta}:(\tilde{\mathcal{H}})^{\beta} \rightarrow \tilde{\mathcal{H}}$ defined analogously to $\epsilon_{\beta}^{\prime}$, one checks easily that

$$
\Omega \circ \tilde{\epsilon}_{\beta}=\epsilon_{\beta}^{\prime} \circ \Omega^{\beta}
$$

Thus if $\tilde{\epsilon}_{\beta}$ is continuous, then also $\epsilon_{\beta}^{\prime}$ is continuous. Now choose $\tilde{G}$ as in Lemma 3 below. Then $\tilde{G}$ satisfies the hypothesis of (5.3), hence $\tilde{\epsilon}_{\beta}$ is continuous. Thus also $\epsilon_{\beta}^{\prime}$ is continuous, and the proof of Theorem 1 is complete.

\section{§5.3. Another group-theoretic Lemma:}


Lemma 3: Each finite group that can be generated by $r-1$ elements $(r \geq 3)$ is the quotient of a finite group $\tilde{G}$ that can be generated by $r-1$ elements and has a subgroup $\tilde{U}$ with the following properties:

(i) $\tilde{U}$ contains no non-trivial normal subgroup of $\tilde{G}$.

(ii) $\tilde{U}$ is self-normalizing in $\tilde{G}$.

(iii) $\operatorname{Aut}(\tilde{G}, \tilde{U})=\operatorname{Inn}(\tilde{G})$.

Proof: Suppose $G$ is a finite group generated by $g_{1}, \ldots, g_{n}$, where $n=r-1 \geq 2$.

Part 1: Reduction to the case that $G$ has trivial center. We need only present $G$ as quotient of a finite group $H$ with trivial center that can also be generated by $n$ elements. This is quite elementary, but we give the argument for completeness. Let $p$ be a prime not dividing the order of $G$. We may assume that the center of $G$ has a non-trivial element $g$. Then $g$ acts non-trivially on the regular $\mathcal{F}_{p}$ module $M$ of $G$ (the group ring over $\mathcal{F}_{p}$ ). The module $M$ is completely reducible by Maschke's theorem. Thus there is an irreducible summand $V$ of $M$ on which $g$ acts non-trivially.

Now consider the semi-direct product $H$ of $G$ and $V$. Let $q=|V|$. There are $q^{n}$ tuples $\left(h_{1}, \ldots, h_{n}\right)$ in $H^{n}$ with $h_{i} \mapsto g_{i}$ for $i=1, \ldots, n$. Since $\mathrm{V}$ is irreducible, the group $\left\langle h_{1}, \ldots, h_{n}\right\rangle$ is either all of $H$, or it is a complement to $V$ in $H$. These complements are all conjugate under $V$ (Schur-Zassenhaus theorem), hence their number is at most $q$. And in each such complement there is a unique lift of the $g_{i}$ 's. Since $n \geq 2$, conclude there is a lift $\left(h_{1}, \ldots, h_{n}\right)$ with $H=<h_{1}, \ldots, h_{n}>$. The center of $H$ maps injectively into the center of $G$, but its image does not contain $g$. Hence $H$ has a center of strictly smaller order than $G$. By induction, this completes the argument.

Part 2: Construction of $\tilde{G}$. From now on assume $G$ has trivial center. Let $S$ be a non-abelian finite simple group with $\operatorname{Aut}(S)=\operatorname{Inn}(S)$, generated by two elements $a, b$, and such that $S$ has a collection of pairwise non-conjugate, self-normalizing proper subgroups $U_{g}$ indexed by the elements $g$ of $G$. For example, take $S$ to be the symplectic group $\mathrm{Sp}_{2 m}(2)$ for large enough $m$, whereby the $U_{g}$ 's can be taken as distinct parabolics containing a common Borel subgroup [Ca; Chapter 11].

Define $\tilde{G}$ to be the semi-direct product $\tilde{G}=A \times{ }^{s} G$ where $A$ is the group of all (not necessarily homomorphic) functions $\alpha: G \rightarrow S$ (with pointwise multiplication) and $G$ acts on $A$ by translation of functions:

$$
{ }^{g} \alpha(h)=\alpha(h g) \quad \text { for } g, h \in G .
$$

Clearly $A \cong S^{m}$, the direct product of $m=|G|$ copies of $S$, and $G$ permutes these factors sharply transitively $(\tilde{G}$ is the wreath product of $G$ and $S$ ). Further, $A$ is minimal normal in $\tilde{G}$, and the centralizer of $A$ in $\tilde{G}$ is trivial. Since distinct minimal normal subgroups centralize each other, $A$ is the unique minimal normal in $\tilde{G}$.

Part 3: Conditions (i)-(iii) hold. We define $\tilde{U}$ as the group of all $\alpha \in A$ with $\alpha(g) \in U_{g}$ for all $g \in G$. As $S$ is simple, condition (i) holds. Since $\tilde{G}$ has trivial center, we can identify $\tilde{G}$ with $\operatorname{Inn}(\tilde{G}) \leq \operatorname{Aut}(\tilde{G})$. We are going to show that $\tilde{U}$ is self-normalizing in $\operatorname{Aut}(\tilde{G})$, which proves (ii) and (iii).

Let $h$ be an element of the normalizer of $\tilde{U}$ in $\operatorname{Aut}(\tilde{G})$. Since $A$ is the unique minimal normal subgroup of $\tilde{G}$, the element $h$ fixes $A$. Recall that the $U_{g}$ are pairwise non-conjugate and self-normalizing in $S \cong \operatorname{Aut}(S)$. From this it follows that $h$ fixes all the simple factors of $A$, and it acts on $A$ as some element of $\tilde{U}$. Thus we may assume that $h$ centralizes $A$. But the centralizer $C$ of $A$ in $\operatorname{Aut}(\tilde{G})$ is normal in $\operatorname{Aut}(\tilde{G})$, and $C \cap \tilde{G}=1$; hence $C$ centralizes $\tilde{G}$, and so $C=1$. Thus $h=1$, and we have proved that $\tilde{U}$ is self-normalizing in $\operatorname{Aut}(\tilde{G})$.

Part 4: $\tilde{G}$ can be generated by $n$ elements. For $i=1, \ldots, n$ define $\alpha_{i} \in A$ by:

$$
\begin{aligned}
\alpha_{i}(1)=a \quad \text { if } i & =1, \quad \alpha_{i}(1)=b \quad \text { if } i>1 \\
\alpha_{i}(g) & =1 \quad \text { for } g \neq 1 .
\end{aligned}
$$

Denote $\left(\alpha_{i}, g_{i}\right)$ by $\tilde{g}_{i}$, and let $H$ be the subgroup of $\tilde{G}$ generated by these elements. We are going to show that $\tilde{G}=H=<\tilde{g}_{1}, \ldots, \tilde{g}_{n}>$. It suffices to show that $A \leq H$. Set $B=A \cap H$. 
For each $i$, let $e_{i}$ be the order of $g_{i}$. Then $\beta_{i} \stackrel{\text { def }}{=} \tilde{g}_{i}^{e_{i}} \in B$, and we have for any $g \in G$ :

$$
\beta_{i}(g)=\alpha_{i}(g) \alpha_{i}\left(g g_{i}\right) \cdots \alpha_{i}\left(g g_{i}^{e_{i}-1}\right) .
$$

This equals $a$ for $i=1$ and $g \in<g_{i}>$; $b$ for $i>1$ and $g \in<g_{i}>$; and 1 if $g \notin<g_{i}>$.

We know there exist elements $\beta \in B$ with $\beta(1) \neq 1$. Among these elements, choose one with the property the set $M=\{g \in G \mid \beta(g) \neq 1\}$ has minimal cardinality. Set $c=\beta(1)$; then $c \neq 1$. Since $S=\langle a, b\rangle$ we may conjugate $\beta$ with products of the $\beta_{i}$ 's to assume that neither of the commutators $[a, c]$ and $[b, c]$ is trivial. Then the function $\beta_{i}^{\prime}=\left[\beta, \beta_{i}\right]$ has $\beta_{i}^{\prime}(1) \neq 1$, and vanishes outside $M$. By the minimality of $M$ it follows that $\beta_{i}^{\prime}(m) \neq 1$ for all $m \in M$. But, $\beta_{i}$, and therefore also $\beta_{i}^{\prime}$, vanishes outside $\left\langle g_{i}\right\rangle$. Thus $M \subset<g_{i}>$ for $i=1, \ldots, n$. Hence $M$ centralizes all $g_{i}$, and therefore lies in the center of $G$. Thus $M=\{1\}$.

We have found $\beta \neq 1$ in $B$ with $\beta(g)=1$ for all $g \neq 1$. Since $S=<a, b>$ it is clear that the conjugates of $\beta$ by products of the $\beta_{i}$ 's generate the full group $\{\alpha \in A: \alpha(g)=1$ for all $g \neq 1\}$. Thus this group lies in $B$. Clearly, the $H$-conjugates of this group generate $A$, which proves $A \leq H$, as claimed. This completes the proof of the Lemma.

\section{A RESULT FOR LATER USE}

The goal of this section is to prove Proposition 3 below. This Proposition is crucial in the paper [FrVo]. Let $G$ be a finite group with trivial center, satisfying the Schur multiplier condition $\left(^{*}\right)$ from $\S 2.2$. Take $U=\{1\}$. All the notation such as $\mathcal{H}^{\mathrm{ab}}=\mathcal{H}_{r}^{\mathrm{ab}}(G, U), \mathcal{E}_{r}^{\mathrm{ab}}, \mathrm{Ni}(\mathbf{C})^{\mathrm{ab}}, \mathcal{H}(\mathbf{C})^{\mathrm{ab}}$ etc. from $\S 1$ refers now to this special case $U=\{1\}$. As usual, $r \geq 3$ is a fixed integer (to be specified later), and $\mathcal{H}^{\text {in }}=\mathcal{H}_{r}^{\text {in }}(G)$.

$\S 6.1$. The automorphisms $\delta_{A}$ of $\mathcal{H}^{\text {in }}$ over $\mathcal{H}^{\text {ab }}$ : This subsection refers only to the (complex) topology of our moduli spaces. Recall that $\mathcal{H}^{\mathrm{ab}}=\mathcal{H}_{r}^{\mathrm{ab}}(G,\{1\})$ is the space of equivalence classes of Galois covers $\chi: X \rightarrow \mathcal{P}^{1}$ with $r$ branch points and with $\operatorname{Aut}\left(X / \mathcal{P}^{1}\right) \cong G$; and $\Lambda: \mathcal{H}^{\text {in }} \rightarrow \mathcal{H}^{\text {ab }}$ is the map sending the class of the pair $(\chi, h)$ to the class of $\chi$. Thus on the fibers over the base point $\boldsymbol{b} \in \mathcal{U}_{r}$, the covering $\Lambda$ induces the canonical map $\mathcal{E}_{r}^{\text {in }} \rightarrow \mathcal{E}_{r}^{\text {ab }}$ sending the class of $\left(\sigma_{1}, \ldots, \sigma_{r}\right)$ modulo $\operatorname{Inn}(G)$ to its class modulo Aut $(G)$ (via the identifications of $\S 1.3)$. It follows that the covering $\Lambda: \mathcal{H}^{\text {in }} \rightarrow \mathcal{H}^{\text {ab }}$ has degree $|\operatorname{Out}(G)|($ where $\operatorname{Out}(G)=$ $\operatorname{Aut}(G) / \operatorname{Inn}(G))$. 
Indeed, the group $\operatorname{Out}(G)$ acts faithfully as a group of automorphisms of the covering $\Lambda$ : For $A \in$ $\operatorname{Aut}(G)$, let $\delta_{A}: \mathcal{H}^{\text {in }} \rightarrow \mathcal{H}^{\text {in }}$ be the map sending $|\chi, h|$ to $|\chi, A h|$. One checks that $\delta_{A}$ is well-defined and continuous. Clearly $\Lambda \circ \delta_{A}=\Lambda$. Thus $\delta_{A}$ is an automorphism of the cover $\Lambda$. Further, $\delta_{A}$ depends only on the class of $A$ modulo $\operatorname{Inn}(G)$. Hence the $\delta_{A}$ yield the desired action of $\operatorname{Out}(G)$.

Let $c$ be the number of conjugacy classes $\neq\{1\}$ of $G$. Suppose that $r=c s$, and that $\mathbf{C}=\left(\mathrm{C}_{1}, \ldots, \mathrm{C}_{r}\right)$ is an $r$-tuple containing each conjugacy class $\neq\{1\}$ of $G$ exactly $s$ times. Since $\operatorname{Aut}(G)$ permutes $\mathrm{C}_{1}, \ldots, \mathrm{C}_{r}$, it follows that $\mathrm{Ni}(\mathbf{C})^{\text {in }}$ is the full inverse image of $\mathrm{Ni}(\mathbf{C})^{\text {ab }}$ under the canonical map $\mathcal{E}_{r}^{\text {in }} \rightarrow \mathcal{E}_{r}^{\text {ab }}$ (see $\left.\S 1.1\right)$. This implies that the Hurwitz space $\mathcal{H}^{\prime}=\mathcal{H}(\mathbf{C})^{\text {in }}$ is the full inverse image of $\mathcal{H}=\mathcal{H}(\mathbf{C})^{\text {ab }}$ under $\Lambda$ (c.f. $\S 1.3$ ). Thus $\Lambda$ restricts to a covering $\Lambda^{\prime}: \mathcal{H}^{\prime} \rightarrow \mathcal{H}$ of degree $|\operatorname{Out}(G)|$, and the group $\operatorname{Out}(G)$ also acts faithfully as a group of automorphisms of this covering. Conclude that if $\mathcal{H}^{\prime}$ and $\mathcal{H}$ are connected then $\Lambda^{\prime}$ is a Galois covering, and the automorphism group of this covering is isomorphic to $\operatorname{Out}(G)$, via the map $A \mapsto \delta_{A}$. From now on, $\delta_{A}$ denotes the restriction of the original map to $\mathcal{H}^{\prime}$.

$\S 6.2$. Choosing suitable Hurwitz spaces: By $\S 2.2$ we can choose $r=c s$ for suitably large $s$ so that each (rational) $r$-tuple $\mathbf{C}$ as in the preceding paragraph has the following property: $\mathrm{Ni}(\mathbf{C})^{\text {in }}$ is non-empty and the Hurwitz group $H_{r}$ acts transitively on this set. Then the Hurwitz spaces $\mathcal{H}^{\prime}=\mathcal{H}(\mathbf{C})^{\text {in }}$ and $\mathcal{H}=\mathcal{H}(\mathbf{C})^{\text {ab }}$ are absolutely irreducible varieties defined over $\mathcal{Q}$, and the covering $\Lambda^{\prime}: \mathcal{H}^{\prime} \rightarrow \mathcal{H}$ is a morphism defined over $\mathcal{Q}$ (by Theorem 1). All complex-analytic automorphisms $\delta_{A}$ of $\Lambda^{\prime}$ are algebraic and defined over $\overline{\mathcal{Q}}$. Indeed, they are even defined over $\mathcal{Q}$ : Namely, for all $\beta \in G_{\mathcal{Q}}$ and $|\chi, h| \in \mathcal{H}^{\prime}$ we have

$$
\begin{aligned}
\left(\delta_{A}\right)^{\beta}\left(|\chi, h|^{\beta}\right)=\left(\delta_{A}(|\chi, h|)\right)^{\beta} & =|\chi, A \circ h|^{\beta}= \\
\left|\chi^{\beta}, A \circ h \circ \beta^{-1}\right| & =\delta_{A}\left(\left|\chi^{\beta}, h \circ \beta^{-1}\right|\right)=\delta_{A}\left(|\chi, h|^{\beta}\right) .
\end{aligned}
$$

It follows that if $\boldsymbol{p}$ is a point of $\mathcal{H}$, rational over some field $k$, and $\boldsymbol{p}^{\prime}$ is a point of $\mathcal{H}^{\prime}$ with $\Lambda^{\prime}\left(\boldsymbol{p}^{\prime}\right)=\boldsymbol{p}$, then the field $k^{\prime}=k\left(\boldsymbol{p}^{\prime}\right)$ is Galois over $k$.

$\S$ 6.3. The main result of $\S$ 6: Fix a point $\boldsymbol{p}^{\prime}=|\chi, h|$ of $\mathcal{H}^{\prime}$, where $\chi: X \rightarrow \mathcal{P}^{1}$ and $h: \operatorname{Aut}\left(X / \mathcal{P}^{1}\right) \rightarrow G$ as usual. Let $\boldsymbol{p}=\Lambda\left(\boldsymbol{p}^{\prime}\right)=|\chi|$ be its image in $\mathcal{H}^{\text {ab }}$, and let $k$ be a field containing $\mathcal{Q}(\boldsymbol{p})$. By Corollary 1 the (Galois) cover $\chi$ can be defined over $k^{\prime}=k\left(\boldsymbol{p}^{\prime}\right)$ so that all its automorphisms are defined over $k^{\prime}$, and $k^{\prime}$ is the minimal extension of $k$ with this property. The resulting function field $L=k^{\prime}(X)$ is Galois over $k^{\prime}(x)=k^{\prime}\left(\mathcal{P}^{1}\right)$, and the group $G_{0} \stackrel{\text { def }}{=} G\left(L / k^{\prime}(x)\right)$ is isomorphic to $G$, via the isomorphism $h_{0}$ which is the composition of the canonical isomorphism $G_{0} \rightarrow \operatorname{Aut}\left(X / \mathcal{P}^{1}\right)$ with $h$. We claim that $L$ is even Galois over $k(x)$.

Indeed, the cover $\chi$ can be defined over $k$ by Corollary 1 . Let $M$ denote the corresponding $k$-form of the field $\bar{L}=\bar{k}(X)$. (The field $M$ is not necessarily contained in $L=k^{\prime}(X)$, thus we do not write $M=k(X)$ ). The field $\bar{L}$ is Galois over $k(x)$, since $k(x)$ is the fixed field of the group of automorphisms of $\bar{L}$ generated by $G(\bar{L} / M)$ and $G(\bar{L} / \bar{k}(x))$. Since $L \cap \bar{k}(x)=k^{\prime}(x)$, elementary Galois theory implies that $G\left(\bar{L} / k^{\prime}(x)\right)$ is the direct product of $G(\bar{L} / \bar{k}(x)) \cong G$ and $G(\bar{L} / L)$. Therefore, as $G$ has trivial center, $G(\bar{L} / L)$ is the centralizer of $N_{1}=G(\bar{L} / \bar{k}(x))$ in $N_{2}=G\left(\bar{L} / k^{\prime}(x)\right)$. From $\S 6.2, k^{\prime}$ is Galois over $k$ and so both $N_{1}$ and $N_{2}$ are normal in $G(\bar{L} / k(x))$. It follows that $G(\bar{L} / L)$ is normal in $G(\bar{L} / k(x))$. Thus $L$ is Galois over $k(x)$, as claimed.

Set $\Omega=G(L / k(x))$. Let $C$ be the centralizer of $G_{0}=G\left(L / k^{\prime}(x)\right)$ in $\Omega$. Then $C$ is normal in $\Omega$, and $C \cap G_{0}=1$ (since $G$ has trivial center). Let $L^{\prime \prime}$ be the fixed field of $C$ in $L$; then $k^{\prime}(x) \cap L^{\prime \prime}=k^{\prime \prime}(x)$ for some field $k^{\prime \prime}$ between $k$ and $k^{\prime}$. It follows that $L^{\prime \prime}$ is a $k^{\prime \prime}$-form of $\bar{L}$ that is Galois over $k^{\prime \prime}(x)$. Thus the cover $\chi$ together with all its automorphisms can be defined over $k^{\prime \prime}$, and so $k^{\prime \prime}=k^{\prime}$ (by minimality of $k^{\prime}$ ). This means $L^{\prime \prime}=L$, hence $C=1$. Thus we have proved that the centralizer of $G_{0}$ in $\Omega$ is trivial. Hence $\Omega$ embeds into $\operatorname{Aut}\left(G_{0}\right)$ (via conjugation action). Thus the above isomorphism $h_{0}: G_{0} \rightarrow G$ induces an injection $h_{1}: \Omega \rightarrow \operatorname{Aut}(G)$. The remaining task is to identify the image of $\Omega$ in $\operatorname{Aut}(G)$. 
Proposition 3: Let $G$ be a finite group with trivial center such that the Schur multiplier of $G$ is generated by commutators (condition (*)). Then the unramified Galois covering $\Lambda^{\prime}: \mathcal{H}^{\prime} \rightarrow \mathcal{H}$ constructed above is a morphism of absolutely irreducible varieties defined over $\mathcal{Q}$, and all automorphisms of this covering are defined over $\mathcal{Q}$. The group $\operatorname{Out}(G)$ acts faithfully on $\mathcal{H}^{\prime}$, inducing the full automorphism group of the covering $\Lambda^{\prime}$; for each $A \in \operatorname{Aut}(G)$, let $\delta_{A}$ denote the automorphism of $\mathcal{H}^{\prime}$ induced by the image of $A$ in Out $(G)$. Then for each point $\boldsymbol{p} \in \mathcal{H}$, rational over some field $k$, and for each point $\boldsymbol{p}^{\prime} \in \mathcal{H}^{\prime}$ with $\Lambda^{\prime}\left(\boldsymbol{p}^{\prime}\right)=\boldsymbol{p}$, there is a Galois extension $L / k^{\prime}(x)$, regular over $k^{\prime}=k\left(\boldsymbol{p}^{\prime}\right)$, such that the following holds:

$L$ is Galois over $k(x)$, and there is an isomorphism $h_{1}$ from $G(L / k(x))$ onto the group $\Delta$ of those $A \in \operatorname{Aut}(G)$ for which $\delta_{A}\left(\boldsymbol{p}^{\prime}\right)$ is conjugate to $\boldsymbol{p}^{\prime}$ under $G\left(k^{\prime} / k\right)$. Furthermore, $h_{1}$ restricts to an isomorphism between $G\left(L / k^{\prime}(x)\right)$ and $\operatorname{Inn}(G)$.

Proof: It only remains to show that $h_{1}(\Omega)=\Delta$ where $h_{1}: \Omega \rightarrow \operatorname{Aut}(G)$ is as defined above. Indeed, it suffices to show that $h_{1}(\Omega) \subset \Delta$ because:

$$
\left|h_{1}(\Omega)\right|=|\Omega|=\left|G_{0}\right| \cdot\left|G\left(k^{\prime}(x) / k(x)\right)\right|=|G| \cdot\left[k^{\prime}: k\right]=|\Delta|
$$

Since $G$ has trivial center, we can (and will) identify $G$ with the $\operatorname{subgroup} \operatorname{Inn}(G)$ of $\operatorname{Aut}(G)$; similarly for $G_{0}$ and for $\bar{G} \stackrel{\text { def }}{=} G\left(\bar{L} / \bar{k}(x)\right.$ ). The map $h_{0}: G_{0} \rightarrow G$ extends naturally to an isomorphism $\operatorname{Aut}\left(G_{0}\right) \rightarrow$ Aut $(G)$, which we again denote by $h_{0}$. The restriction map $\bar{G} \rightarrow G_{0}$ is an isomorphism; let $R:$ Aut $(\bar{G}) \rightarrow$ $\operatorname{Aut}\left(G_{0}\right)$ be its natural extension. Define the isomorphism $\bar{h}: \operatorname{Aut}(\bar{G}) \rightarrow \operatorname{Aut}(G)$ by $\bar{h}=h_{0} \circ R$. Clearly, the image $\bar{\Omega}$ of $G(\bar{L} / k(x))$ in Aut $(\bar{G})$ (via conjugation action) corresponds under $R$ to the image of $\Omega=$ $G(L / k(x))$ in $\operatorname{Aut}\left(G_{0}\right)$. Thus $h_{1}(\Omega)=h_{0} R(\bar{\Omega})=\bar{h}(\bar{\Omega})$. From the previous paragraph, it remains to show that $\bar{h}(\bar{\Omega}) \subset \Delta$.

From the definitions, $\bar{h}$ is induced from the map $\bar{G} \rightarrow G$ that is the composition of the canonical isomorphism $\bar{G} \rightarrow \operatorname{Aut}\left(X / \mathcal{P}^{1}\right)$ with $h$. Thereby, this canonical isomorphism is explicitly given as follows: It sends $B^{*}$ to $B$, where $B$ is any element of $\operatorname{Aut}\left(X / \mathcal{P}^{1}\right)$ and $B^{*} \in \bar{G}=G(\bar{L} / \bar{k}(x))$ is defined by $B^{*}(f)=f \circ B^{-1}$ (pulling back of functions), for $f \in \bar{L}=\bar{k}(X)$. Therefore:

(7) $\bar{h}\left(B^{*}\right)=h(B)$ for all $B \in \operatorname{Aut}\left(X / \mathcal{P}^{1}\right)$.

Now let $\bar{\alpha}$ be an element of $\bar{\Omega}$, and let $\alpha \in G(\bar{L} / k(x))$ be a pre-image of $\bar{\alpha}$. Let $\beta \in G(\bar{k} / k)$ be the image of $\alpha^{-1}$ under $G(\bar{L} / k(x)) \rightarrow G(\bar{k}(x) / k(x)) \cong G(\bar{k} / k)$ (where the first map is restriction). We claim that the element $A=\bar{h}(\bar{\alpha})$ of $\operatorname{Aut}(G)$ satisfies

$$
\delta_{A}\left(\boldsymbol{p}^{\prime}\right)=\left(\boldsymbol{p}^{\prime}\right)^{\beta} .
$$

This means that $A=\bar{h}(\bar{\alpha})$ lies in $\Delta$, and $\bar{h}(\bar{\Omega}) \subset \Delta$ as desired. Thus it only remains to prove (8).

By Corollary 1(a) the cover $\chi: X \rightarrow \mathcal{P}^{1}$ can be defined over $k$. This is compatible with the $\bar{k}$-structure, but not necessarily with the $k^{\prime}$-structure on $X$ considered above. However, the remainder of the proof does not refer to this $k^{\prime}$-structure anymore, so we assume now that $X$ and $\chi$ are defined over $k$. This yields an action of $G(\bar{k} / k)$ on $\bar{L}=\bar{k}(X)$, denoted $f \mapsto f^{\sigma}(f \in \bar{L}, \sigma \in G(\bar{k} / k))$. The map $f \mapsto f^{\sigma}$ acts on the subfield $\bar{k}(x)$ in the natural way (i.e., through the canonical isomorphism $G(\bar{k}(x) / k(x)) \rightarrow G(\bar{k} / k)$ ), since $\chi$ is defined over $k$. Thus the map $f \mapsto f^{\beta}$ and the map $\alpha^{-1} \in G(\bar{L} / k(x))$ restrict to the same element of $G(\bar{k}(x) / k(x))$, and so there is some $D \in G(\bar{L} / \bar{k}(x))=\bar{G}$ with $f^{\beta}=D \alpha^{-1}(f)$ for all $f \in \bar{L}$. Replacing $\alpha$ by $\alpha D^{-1}$ changes $A=\bar{h}(\bar{\alpha})$ only by the inner automorphism $\bar{h}\left(D^{-1}\right)$ of $G$, and the map $\delta_{A}$ remains unchanged. Thus we may assume $D=1$, so that $f^{\beta}=\alpha^{-1}(f)$ for all $f \in \bar{L}$. Then we have for all $B \in \operatorname{Aut}\left(X / \mathcal{P}^{1}\right)$, $f \in \bar{L}:$

$$
\left(B^{\beta^{-1}}\right)^{*}(f)=f \circ\left(B^{-1}\right)^{\beta^{-1}}=\left[f^{\beta} \circ B^{-1}\right]^{\beta^{-1}}=\alpha B^{*} \alpha^{-1}(f)=\bar{\alpha}\left(B^{*}\right)(f)
$$

hence

$$
\left(B^{\beta^{-1}}\right)^{*}=\bar{\alpha}\left(B^{*}\right)
$$

Finally, Theorem 1 gives $\left(\boldsymbol{p}^{\prime}\right)^{\beta}=|\chi, h|^{\beta}=\left|\chi^{\beta}, h \circ \beta^{-1}\right|=\left|\chi, h \circ \beta^{-1}\right|$, where $h \circ \beta^{-1}: \operatorname{Aut}\left(X / \mathcal{P}^{1}\right) \rightarrow G$ is the isomorphism sending $B$ to $h\left(B^{\beta^{-1}}\right)$. By (7) and (9) we have

$$
h\left(B^{\beta^{-1}}\right)=\bar{h}\left(\left(B^{\beta^{-1}}\right)^{*}\right)=\bar{h}\left(\bar{\alpha}\left(B^{*}\right)\right)=\bar{h}(\bar{\alpha})\left(\bar{h}\left(B^{*}\right)\right)=\bar{h}(\bar{\alpha})(h(B))=A \circ h(B)
$$

Thus $\left(\boldsymbol{p}^{\prime}\right)^{\beta}=|\chi, A \circ h|=\delta_{A}\left(\boldsymbol{p}^{\prime}\right)$, which proves (8). This completes the proof of Proposition 3 . 


\section{APPENDIX}

This is a slightly modified exposition on a result of Conway and Parker [CP]. We only consider the case that the union $S$ of conjugacy classes of $G$ that occurs in $[\mathrm{CP}]$ is all of $G \backslash\{1\}$, and that $M(G)$ is generated by commutators. This is what is needed above. The restriction to this special case allows for some simplifications. Further, we correct some errors from $[\mathrm{CP}]$.

A. Introduction: Let $G$ be a finite group, and let $r \geq 3$ be an integer. For $i=1, \ldots, r-1$ let $Q_{i}: G^{r} \rightarrow G^{r}$ be given by expression (2) of $\S 1.3$.

The Hurwitz class of $\boldsymbol{\sigma}=\left(\sigma_{1}, \ldots, \sigma_{r}\right) \in G^{r}$ is defined to be the orbit of $\boldsymbol{\sigma}$ under the group generated by $Q_{1}, \ldots, Q_{r-1}$. Clearly, the evaluation function $E(\boldsymbol{\sigma})=\sigma_{1} \cdots \sigma_{r}$ is constant on Hurwitz classes. The same is true for the shape function $S(\boldsymbol{\sigma})=\left(n_{C}(\boldsymbol{\sigma})\right)_{\mathrm{C}}$, which maps $\boldsymbol{\sigma}$ to the shape vector containing the numbers $n_{C}(\boldsymbol{\sigma})=\#\left\{i: \sigma_{i} \in \mathrm{C}\right\}$, where $\mathrm{C}$ runs through the set of conjugacy classes of $G$. In certain cases, these functions form a complete set of invariants for the Hurwitz classes:

Theorem (Conway-Parker): Suppose the Schur multiplier $M(G)$ is generated by commutators (as defined in §2.4). Then there exists an integer $N$ with the following property: If $\boldsymbol{\sigma}=\left(\sigma_{1}, \ldots, \sigma_{r}\right) \in G^{r}$ satisfies $n_{C}(\boldsymbol{\sigma}) \geq$ $N$ for all conjugacy classes $\mathrm{C} \neq\{1\}$ of $G$, then the Hurwitz class of $\boldsymbol{\sigma}$ consists of all $\boldsymbol{\tau}=\left(\tau_{1}, \ldots, \tau_{r}\right) \in G^{r}$ with $\tau_{1} \cdots \tau_{r}=\sigma_{1} \cdots \sigma_{r}$ and $S(\boldsymbol{\tau})=S(\boldsymbol{\sigma})$.

In the remainder of this Appendix we give a proof of this theorem, adapted from [CP].

B. Central Extensions: Let $\varphi: \hat{G} \rightarrow G$ be a central extension (of groups), such that $\hat{G}$ is generated by elements $\hat{a}(a \in G)$ with $\varphi(\hat{a})=a$. Use the notation $a^{b}=b^{-1} a b$. We assume that the following relations hold:

$$
\hat{a} \hat{b}=\hat{b} \widehat{a^{b}}
$$

Proposition: Suppose $M(G)$ is generated by commutators. If $\boldsymbol{\sigma}=\left(\sigma_{1}, \ldots, \sigma_{r}\right)$, $\boldsymbol{\tau}=\left(\tau_{1}, \ldots, \tau_{r}\right) \in G^{r}$ satisfy $\sigma_{1} \cdots \sigma_{r}=\tau_{1} \cdots \tau_{r}$ and $S(\boldsymbol{\sigma})=S(\boldsymbol{\tau})$, then $\hat{\sigma}_{1} \cdots \hat{\sigma}_{r}=\hat{\tau}_{1} \cdots \hat{\tau}_{r}$.

Proof: Let $\mathcal{F}$ be the free group on generators $\bar{a}(a \in G)$, let $\mathcal{R}$ be the kernel of the homomorphism $\mathcal{F} \rightarrow G$ sending $\bar{a}$ to $a$, and let $\psi: \mathcal{F} \rightarrow \hat{G}$ be the homomorphism sending $\bar{a}$ to $\hat{a}$. We prove:

$$
\psi(\mathcal{R} \cap[\mathcal{F}, \mathcal{F}])=1
$$

Since $\psi(\mathcal{R}) \leq \operatorname{ker}(\varphi) \leq Z(\hat{G})$, the center of $\hat{G}$, clearly $\psi([\mathcal{R}, \mathcal{F}])=1$. By the general theory of the Schur multiplier (e.g., [Hu; p. 631]) the quotient of $\mathcal{R} \cap[\mathcal{F}, \mathcal{F}]$ by $[\mathcal{R}, \mathcal{F}]$ is isomorphic to the Schur multiplier $M(G)$. The hypothesis that $M(G)$ is generated by commutators means that the group $\mathcal{R} \cap[\mathcal{F}, \mathcal{F}]$ is generated modulo $[\mathcal{R}, \mathcal{F}]$ by commutators $z=[x, y], x, y \in \mathcal{F}$. Since $a=\varphi \circ \psi(x)$ and $b=\varphi \circ \psi(y)$ commute in $G$, the relations (I) imply that $\hat{a}$ and $\hat{b}$ commute in $\hat{G}$. Since $\psi(x) \in \hat{a} Z(\hat{G})$ and $\psi(y) \in \hat{b} Z(\hat{G})$, we get $\psi(z)=[\psi(x), \psi(y)]=[\hat{a}, \hat{b}]=1$. This proves $(\mathrm{II})$.

¿From (II) the map $\psi: \mathcal{F} \rightarrow \hat{G}$ induces $\bar{\psi}: \overline{\mathcal{F}} \rightarrow \hat{G}$, where $\overline{\mathcal{F}}=\mathcal{F} /(\mathcal{R} \cap[\mathcal{F}, \mathcal{F}])$. Clearly $\overline{\mathcal{R}} \cap[\overline{\mathcal{F}}, \overline{\mathcal{F}}]=1$, where $\overline{\mathcal{R}}$ is the image of $\mathcal{R}$ in $\overline{\mathcal{F}}$. The kernel of $\bar{\psi}$ lies in $\overline{\mathcal{R}}$, hence $\bar{\psi}(\overline{\mathcal{R}}) \cap[\hat{G}, \hat{G}]=1$. Let $A$ be the abelian group $\hat{G} /[\hat{G}, \hat{G}]$. Since $\bar{\psi}(\overline{\mathcal{R}})=\operatorname{ker}(\varphi)$, it follows that $\hat{G}$ embeds as a subgroup of $G \times A$, via the map that sends $g \in \hat{G}$ to $(\varphi(g), g[\hat{G}, \hat{G}])$.

Viewing $\hat{G}$ as a subgroup of $G \times A$ via this embedding, we have $\hat{a}=\left(a, t_{a}\right)$ for each $a \in G$, where $t_{a} \in A$. The relations (I) yield $t_{a}=t_{a^{b}}$ for all $a, b \in G$. Thus $t_{a}$ depends only on the conjugacy class of $a$. Now the Proposition follows:

$$
\prod \hat{\sigma}_{i}=\prod\left(\sigma_{i}, t_{\sigma_{i}}\right)=\left(\prod \sigma_{i}, \prod t_{\sigma_{i}}\right)=\left(\prod \tau_{i}, \prod t_{\tau_{i}}\right)=\prod \hat{\tau}_{i}
$$

The final section produces an equivalence relation on the semigroup of Hurwitz classes of arrays of elements of $G$. The quotient by this equivalence relation turns out to be a group $\hat{G}$, satisfying the hypotheses of the Proposition. 
C. Congruence Classes of Words: Consider the semi-group of words in the symbols $\tilde{a}(a \in G \backslash\{1\})$, under concatenation. Define the Hurwitz class $H(w)$ of a word $w=\tilde{a}_{1} \cdots \tilde{a}_{r}$ to be the set of all words that can be obtained from $w$ by iteration of the operations $Q_{i}$ given by expression (2) of $\S 1.3$ (where we now write $\tilde{a}_{1} \cdots \tilde{a}_{r}$ instead of $\left.\left(a_{1}, \ldots, a_{r}\right)\right)$. By abuse of notation, we will denote the Hurwitz class $H(\tilde{a})=\{\tilde{a}\}$ again by $\tilde{a}$. Denote the set of Hurwitz classes by $\mathcal{H}=\mathcal{H}(G)$. The multiplication $H\left(w_{1}\right) H\left(w_{2}\right)=H\left(w_{1} w_{2}\right)$ is well-defined and makes $\mathcal{H}$ into a semi-group, generated by the elements $\tilde{a}$ ( $a \in G \backslash\{1\}$ ). They satisfy the relations

$$
\tilde{a} \tilde{b}=\tilde{b} \widetilde{a^{b}}
$$

We fix the notation $A=\tilde{a}_{1} \cdots \tilde{a}_{r}, B=\tilde{b}_{1} \cdots \tilde{b}_{s}$ for general elements of $\mathcal{H}$. As in the Introduction, we have the evaluation function $E(A)=a_{1} \cdots a_{r}$ and the shape function $S(A)$ on Hurwitz classes; both are semi-group homomorphisms.

Lemma 1: If $E(A)=1$ then $A \in Z(\mathcal{H})$.

Proof: For $b \in G$ we have $\tilde{b} A=\tilde{b} \tilde{a}_{1} \cdots \tilde{a}_{r}=\widetilde{a_{1}} \widetilde{b^{a_{1}}} \tilde{a}_{2} \cdots \tilde{a}_{r}=\tilde{a}_{1} \tilde{a}_{2} b^{\widetilde{a_{1} a_{2}}} \tilde{a}_{3} \cdots \tilde{a}_{r}=\cdots=A b^{a_{1}} \widetilde{a_{2} \cdots a_{r}}=A \tilde{b}$. Since $\mathcal{H}$ is generated by the $\tilde{b}$, the claim follows.

Let $o(a)$ denote the order of an element $a \in G$. Set

$$
U=\prod_{a \in G \backslash\{1\}} \tilde{a}^{o(a)}
$$

The factors in this product are in the center of $\mathcal{H}$ by Lemma 1 . Therefore the ordering in the product does not matter. Then also $U$ lies in the center of $\mathcal{H}$, and for any $b \in G \backslash\{1\}$, we can write $U$ in the form $U=\tilde{b} A=A \tilde{b}$ for some $A$. Set $A \equiv B$ iff there exist integers $n, k \geq 0$ with $U^{n} A=U^{k} B$. It is easy to check that this yields a congruence relation on the semi-group $\mathcal{H}$. We let $\hat{G}$ denote the quotient of $\mathcal{H}$ by this congruence relation. The following Lemma allows us to apply the results of the previous section.

Lemma 2: The semigroup $\hat{G}$ is in fact a group, which is a central extension of $G$ via the map $\varphi: \hat{G} \rightarrow G$ induced by the evaluation function $E$. If we let $\hat{a}$ denote the image of $\tilde{a}$ in $\hat{G}$, then $\varphi(\hat{a})=a$, the relations (I) hold and the elements $\hat{a}$ generate $\hat{G}$.

Proof: Lemma 1 shows that the semi-group $\hat{G}$ is a central extension of $G$ via $\varphi$. It only remains to show that $\hat{G}$ is in fact a group, the other assertions are clear.

For any $b \in G \backslash\{1\}$ we have $U=\tilde{b} A=A \tilde{b}$ for some $A$, by the above remark. Thus $\hat{b}$ is invertible in $\hat{G}$. Since the $\hat{b}$ generate $\hat{G}$, all elements of $\hat{G}$ are invertible, and $\hat{G}$ is a group.

Lemma 3: If $g, h \in G$ are conjugate elements of order $m>1$, and $G=<a_{1}, \ldots, a_{r}>$, then $\tilde{g}^{m} \tilde{a}_{1} \cdots \tilde{a}_{r}=$ $\tilde{h}^{m} \tilde{a}_{1} \cdots \tilde{a}_{r}$.

Proof: By Lemma 1 we have $\tilde{g}^{m} \in Z(\mathcal{H})$. Hence

$$
\begin{aligned}
\tilde{g}^{m} \tilde{a}_{1} \cdots \tilde{a}_{r}=\tilde{a}_{1} \cdots \tilde{a}_{j-1} \tilde{g}^{m} \tilde{a}_{j} \cdots \tilde{a}_{r} & =\tilde{a}_{1} \cdots \tilde{a}_{j-1} \tilde{g}^{m-1} \tilde{a}_{j}{\widetilde{g^{a_{j}}}}_{\tilde{a}_{j+1}} \cdots \tilde{a}_{r} \\
=\cdots & =\tilde{a}_{1} \cdots \tilde{a}_{j-1} \tilde{a}_{j}\left(\widetilde{g^{a_{j}}}\right)^{m} \tilde{a}_{j+1} \cdots \tilde{a}_{r}=\left(\widetilde{g^{a_{j}}}\right)^{m} \tilde{a}_{1} \cdots \tilde{a}_{r} .
\end{aligned}
$$

Since $a_{1}, \ldots, a_{r}$ generate the finite group $G$, the claim follows by induction.

Lemma 4: If $S(B) \geq S(X U)$ (componentwise) for some $X \in \mathcal{H}$ then $B=X V$ for some $V \in \mathcal{H}$. In particular, if $S(B) \geq S\left(U^{2}\right)$ then $B=U V$ for some $V \in \mathcal{H}$.

Proof: By induction it suffices to consider the case $X=\tilde{a}$. Let $m$ be the order of $a$, and $\Gamma$ the conjugacy class of $a\left(\right.$ in $\left._{\tilde{b}} G\right)$. Since $S(B) \geq S(\tilde{a} U)$, there are more than $m|\Gamma|$ indices $i=1, \ldots, s$ for which $b_{i} \in \Gamma$ (where $B=\tilde{b}_{1} \cdots \tilde{b}_{s}$ ). Hence there is some $g \in \Gamma$ such that $b_{i}=g$ for more than $m$ indices $i$. Use (I') to conclude that $B$ can be written as $B=\tilde{g}^{m} \tilde{w}_{1} \cdots \tilde{w}_{k}$ with $w_{1}=g$. Then each nontrivial conjugacy class of $G$ contains some $w_{i}$. Therefore the union of the conjugates of the subgroup $\left\langle w_{1}, \ldots, w_{k}\right\rangle$ is all of $G$. It is well-known that this implies that $G=<w_{1}, \ldots, w_{k}>$ (e.g., [FrJ, 12.4]). Thus Lemma 3 yields $B=\tilde{g}^{m} \tilde{w}_{1} \cdots \tilde{w}_{k}=\tilde{a}^{m} \tilde{w}_{1} \cdots \tilde{w}_{k}=\tilde{a} V$ for some $V$.

Let $\mathcal{H}_{i}$ be the set of all $V \in \mathcal{H}$ with $S(V)=S\left(U^{i}\right)$. By Lemma 4 the map $\mathcal{H}_{i} \rightarrow \mathcal{H}_{i+1}, V \mapsto U V$, is surjective for $i \geq 1$. Since the $\mathcal{H}_{i}$ are finite sets, there must be some integer $K$ such that for all $i \geq K$ this map is bijective. Thus for $A, B \in \mathcal{H}_{K}$ and $n \geq 0$ the relation $U^{n} A=U^{n} B$ implies $A=B$. 
Conclusion: Suppose $M(G)$ is generated by commutators. Let $A=\tilde{a}_{1} \cdots \tilde{a}_{r}, B=\tilde{b}_{1} \cdots \tilde{b}_{r}$ be elements of $\mathcal{H}$ with $S(A)=S(B) \geq S\left(U^{K}\right)$ and $a_{1} \cdots a_{r}=b_{1} \cdots b_{r}$. Then $A=B$. That is, $\left(b_{1}, \ldots, b_{r}\right)$ is in the Hurwitz class of $\left(a_{1}, \ldots, a_{r}\right)$.

Proof: By Lemma 2 and the Proposition of $\S \mathrm{B}$, we have $\prod \hat{a}_{i}=\prod \hat{b}_{i}$. Hence $U^{n} A=U^{k} B$ for some $n, k \geq 0$. Since $S(A)=S(B)$ we have $n=k$. By Lemma 4 we have $A=X W, B=X V$ for some $V, W \in \mathcal{H}_{K}, X \in \mathcal{H}$. Since $\hat{G}$ is a group, we have $Y X=U^{t}$ for some $Y \in \mathcal{H}, t \geq 0$.

Summarizing, we have $U^{n} A=U^{n} B$, hence $U^{n} X W=U^{n} X V$. Multiplying both sides by $Y$ from the left we get $U^{n+t} W=U^{n+t} V$. Since $W, V \in \mathcal{H}_{K}$ this implies $W=V$ by the remarks after Lemma 4 . Thus $A=X W=X V=B . \quad \square$

The Theorem of $\S$ A now follows from this Conclusion.

\section{Bibliography}

[Ax] J. Ax, The elementary theory of finite fields, Annals of Math. 88 (1968), 239-271.

[BF] R. Biggers and M. Fried, Moduli Spaces of Covers of $\mathcal{P}^{1}$ and the Hurwitz Monodromy Group, J. für die reine und angew. Math. 335 (1982), 87-121.

[C] R. Carter, Simple Groups of Lie Type, John Wiley and Sons, Pure and Applied Mathematics XXVIII (1972).

[Cl] A. Clebsch, Zur Theorie der Riemann'schen Fläche, Math. Annalen 6 (1872), 216-230.

[CP] J. H. Conway and R. A. Parker, On the Hurwitz number of arrays of group elements, unpublished preprint, September 1988.

[CHa] K. Coombs and D. Harbater, Hurwitz families and arithmetic Galois groups, Duke Math. J. 52 (1985), 821-839.

[DFr] P. Debes and M. Fried, Regular extensions of $\mathcal{R}(x)$ and Rigidity, preprint.

[Fr, 1] M. Fried, Fields of definition of function fields and Hurwitz families - groups as Galois groups, Comm. in Algebra 5(1) (1977), 17-82.

[Fr, 2] M. Fried, Galois groups and Complex Multiplication, TAMS 235 (1978), 141-162.

[Fr, 3] M. Fried, Arithmetic of 3 and 4 branch point covers: a bridge provided by non-congruence subgroups of $\mathrm{SL}_{2}(\mathcal{Z})$, Progress in Math. Birkhäuser 81 (1989), 77-117.

[FrJ] M. Fried and M. Jarden, Field Arithmetic, Springer-Ergebnisse 11 (1986).

[FrJ, 2] M. Fried and M. Jarden, Diophantine properties of subfields of $\mathcal{Q}$, Amer. J. Math. 100 (1978), 653666.

[FrVo] M. Fried and H. Völklein, The embedding problem over a Hilbertian PAC-field, preprint.

[Fu] W. Fulton, Hurwitz schemes and irreducibility of moduli of algebraic curves, Annals of Math. 90 (1969), 542-575.

[Fy] G. Frey, Pseudo-algebraically closed fields with non-archimedean real valuations, J. of Algebra 26 (1973), 202-207.

[Hu] B. Huppert, Endliche Gruppen I, Graduate Texts-Springer (1967), Berlin, Heidelberg, New York.

[Hur] A. Hurwitz, Über Riemann'sche Flächen mit gegebenen Verzweigungspunkten, Math. Annalen 39 (1891), 1-61.

[LD] A. Lubotzky and L. v. d. Dries, Subgroups of free profinite groups and large subfields of $\overline{\mathcal{Q}}$, Israel J. Math. 39 (1981), 25-45.

[Ma,1] H. Matzat, Konstruktive Galoistheorie, Lecture Notes in Math. 1284, Springer Verlag (1986).

[Ma,2] H. Matzat, Zöpfe und Galois'sche Gruppen, preprint 1990.

$[\mathrm{P}]$ F. Pop, The totally real numbers are PRC, preprint as of Oct. '90.

[Se] J.-P. Serre, Topics in Galois Theory, notes by H. Darmon, Harvard 1989.

[SGA1] A. Grothendieck, Revêtements étale et groupes fondamental, Lecture Notes in Math. 224, Springer Verlag (1971).

[Th] J.G. Thompson, Some finite groups which occur as $\operatorname{Gal}(L / K)$ where $K \leq \mathcal{Q}\left(\mu_{n}\right)$, J. of Algebra 89 (1984), 437-499.

[Vo] H. Völklein, $\mathrm{PSL}_{2}(q)$ and extensions of $\mathcal{Q}(x)$, BAMS 24 (1991), 145-153.

[W] A. Weil, The field of definition of a variety, Amer. J. Math 78 (1956), 509-524. 
[Ws] R. Weissauer, Der Hilbertsche Irreduzibilitätssatz, J. für die reine und angew. Math. 334 (1982), 203-220.

Mike Fried

Department of Mathematics

UC Irvine

Irvine, California 92717
Helmut Völklein

Department of Mathematics

University of Florida

Gainesville, Fl 32611 